\title{
Vortex Actuation via Electric Ducted Fans: an Experimental Study
}

\author{
George Andrikopoulos ${ }^{1}$ [D . George Nikolakopoulos ${ }^{1}$
}

Received: 5 April 2018 / Accepted: 21 August 2018 / Published online: 4 September 2018

(C) The Author(s) 2018

\begin{abstract}
The presented work investigates the potential of utilizing commercially available Electric Ducted Fans (EDFs) as adhesion actuators, while providing a novel insight on the analysis of the adhesion nature related to negative pressure and thrust force generation against a target surface. To this goal, a novel EDF-based Vortex Actuation Setup (VAS) is proposed for monitoring important properties such as adhesion force, pressure distribution, current draw, motor temperature etc. during the VAS' operation when placed in variable distances from a test surface. In addition, this work is contributing towards the novel evaluation of different design variables and modifications to original EDF structures, with the goal of analyzing their effect on the prototype VAS, while optimizing its adhesion efficiency for its future incorporation in a wall-climbing robot for inspection and repair purposes.
\end{abstract}

Keywords Electric Ducted Fans · Vortex Actuation · Negative pressure · Adhesion force

\section{Introduction}

EDFs have been traditionally popular for their utilization as a propulsion method for both big and small-scale aircrafts, mainly due to an increased thrust efficiency by the ducts reduction of tip vortices and the pressure drops at the blade tips, when compared to their open-air equivalents $[1,2]$. Typical EDF designs, as graphically displayed in Fig. 1, consist of a motor and an impeller, encased by a cylindrical duct and shroud. A rotor and aft cone are incorporated to reduce the turbulence of the air flow through the duct around the motor (Fig. 1a), although the aft cone is omitted in cases of high-power motors (Fig. 1b) due to their cooling limitation from the generated air flow. In the latter cases, the duct has been traditionally longer in height for fully enclosing the motor and thus decreasing any large air volume changes for optimizing airflow.

From an application point of view, while the EDFs have been utilized mostly for propelling a craft, their ability to generate a negative pressure and actively adhere to a surface,

George Andrikopoulos

geoand@1tu.se

George Nikolakopoulos

geonik@ltu.se

1 Control Engineering Group, Luleå University of Technology, SE-97187 Luleå, Sweden when their ducted structure is placed at a close proximity [3, 4], has led to different application areas, with the most recent being the Wall-Climbing Robots (WCRs) [5]. Although the WCRs presented in the related literature have been utilizing a number of different methods for adhesion, from passive $[6,7]$ and active suction cups $[8,9]$ to vortex chambers $[3,10]$ magnetic attraction $[11,12]$ and oscillated propulsion [13, 14] the use of an EDF-based design as an adhesion method, provides important advantages that could increase the potential usability of WCRs, advantages that have not been addressed yet at full extent.

Specifically, EDFs do not require the adhesion mechanism to be in contact with the target surface, which alleviates the design challenges of adhesion in cases of curved, rough or non-magnetic target surfaces [3]. In addition, such a design technique has an impact on the overall cost, since no external equipment (e.g. compressor, filters, tubes etc.) must be used for maintaining adhesion, which produces an untethered-friendly solution.

In the small number of applications found in related literature that utilize an EDF-based design, the main research aspect has been either the negative pressure generation [3] or thrust $[4,15]$ generated by the EDF. From this related works it becomes apparent that the experimental evaluation has been constrained on investigating the effect of the gap, i.e. the distance of the EDF shroud to the target surface, on the adhesion efficiency. Thus, there is an identified gap on the investigation of additional important 
Fig. 1 Typical EDF designs in assembled and exploded view

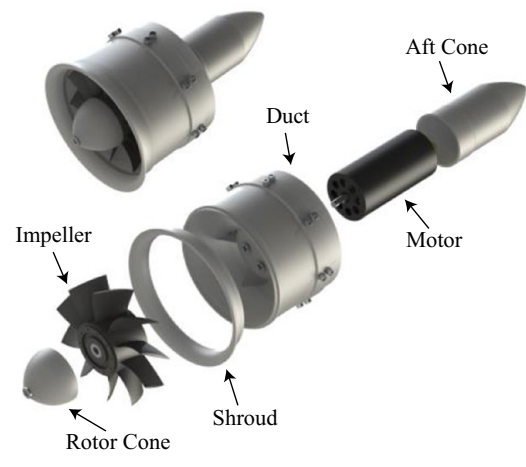

(a)

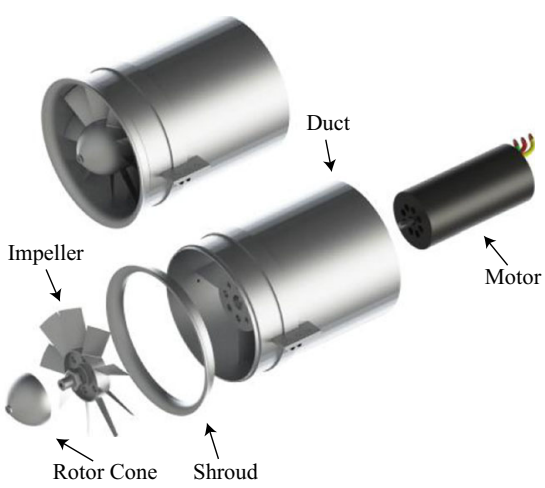

(b) design parameters, such as the EDF's structural placement, the length and dimensions of the duct, the dimensions of the shroud and its distance from the surface, which complicates the modeling and control of such a system.

In addition, the general lack of a theoretical modeling analysis describing the connection between the negative pressure and generated thrust, the properties of the adhesion force and its connection to the generated pressure vortex, or advanced algorithms for the control of these variables, increase the importance of initially identifying the most important design parameters, evaluating their effect and finally implementing more advanced models and control algorithms. Also, aerodynamic simulations of such systems have been traditionally characterized by heavy computational load, especially when intricate constraints are introduced in the designs, while the required number of iterations needed to investigate their operation under a large set of different design properties further increased the difficulty of analyzing them from a multi-parameterized mathematical perspective.

In the presented case, an experimental investigation of these complicated phenomena is pursued and to this goal, modern fast prototyping techniques (3D printing) are used to develop a novel low-cost and low-complexity prototype, accompanied by a novel methodology for experimentally evaluating the proposed VAS for different EDF cases in an accurate and safe manner. Thus, the main novelty of this article stems from the design, development and evaluation of a novel adhesion system with the goal of investigating the potential of utilizing commercially available Electric Ducted Fans (EDFs) as adhesion actuators. A novel insight on the analysis of the adhesion nature related to negative pressure and thrust force generation against a target surface is presented in detail and through the prism of various structural modifications to the original EDF designs. The ultimate objective of this work is to mechanically optimize the VAS actuation efficiency and provide novel considerations on future designs of WCRs incorporating the particular actuator.
The rest of this article is structured as follows. Section 2 covers the fundamental negative pressure adhesion principles, while Section 3 presents the conceptual design of the proposed VAS, the selected design variables, the prototype implementation of the setup and the utilized components. In Section 4, the experimental evaluation of the VAS is presented in detail from the prism of the design modifications, while insight is being provided on the system's properties and the optimization of its adhesion efficiency. Finally, the concluding remarks are provided in Section 5.

\section{Negative Pressure Adhesion Principle}

Negative pressure adhesion works on the principle of generating and maintaining a low-pressure zone $P_{a}$ inside a cavity compared to the surrounding outside pressure $P_{b}$. As depicted in Fig. 2, the difference in pressure will induce a force $F_{S}$ across the projected cavity area onto the target surface, $A$, by the high-pressure region [16] as:

$F_{s}=A\left(P_{b}-P_{a}\right)$.

Considering an EDF-based adhesion mechanism, the lowpressure zone lies within its shroud, hence the active pressure area is $A=\pi\left(r_{s}^{2}-r_{d}^{2}\right) \mathrm{mm}^{2}$ with $r_{s}$ and $r_{d}$ denoting the outer shroud radius and the duct's internal radius, respectively. In this definition, the effect of the shroud's curvature is neglected for simplification purposes,

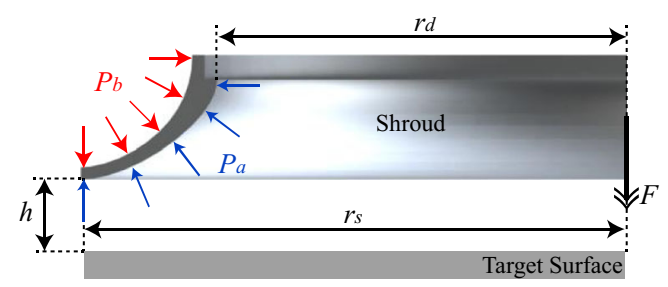

Fig. 2 Shroud detail in half-section view with highlighted pressure zones and basic geometrical properties 
while the area $A$ will from now on be referred to as the active area.

In this configuration, an increase in applied force can occur via a decrease in the shroud's pressure, or an increase in the active area. Furthermore, air flow through the lowpressure zone $P_{a}$ is introduced by the distance between the shroud's end and the test surface, $h$, as displayed in Fig. 2. The flows $Q_{i n}$ and $Q_{\text {out }}$ entering and exiting the chamber, respectively, are defined by,

$Q_{\text {in }}=A_{\text {in }} u_{\text {in }}, Q_{\text {out }}=A_{\text {out }} u_{\text {out }}$

where $A_{\text {in }}$ and $A_{\text {out }}$ denote the in- and outlet areas (Fig. 3), while $u_{\text {in }}$ and $u_{\text {out }}$ denote the air velocity into and out of these areas. The outlet area, also known as Fan Swept Area (FSA), is defined as $A_{\text {out }}=\pi\left(r_{d}^{2}-r_{m}^{2}\right) \mathrm{mm}^{2}$, with $r_{m}$ denoting the radius of the motor's cylindrical case, while the inlet area $A_{i n}=2 \pi h r_{s} \mathrm{~mm}^{2}$ is proportional to the gap height $h$ and the shroud radius $r_{s}$.

For a negative pressure zone to be generated, the flow out of the chamber must be higher than the flow in, for a specific period. As $P_{a}$ lowers, air is pulled in through the gap and after a time $t$ the flow reaches an equilibrium, with the lower pressure zone being maintained. The steady state operational point is defined as:

$A_{\text {in }} u_{\text {in }}=A_{\text {out }} u_{\text {out }}$

In other words, a mass flow imbalance occurs for an increase in the EDF's rotational speed. As air is drawn out from $A_{\text {out }}$ at a faster rate than in through $A_{\text {in }}$, this results in an additional pressure decrease in the inlet area, which is defined as the air volume constrained by the shroud and its projection to the target surface. This decrease in pressure will speed up the air flowing in through the gap until a steady-state is reached, while as the rotational speed is kept constant, the negative pressure and flow rates remain constant as well.
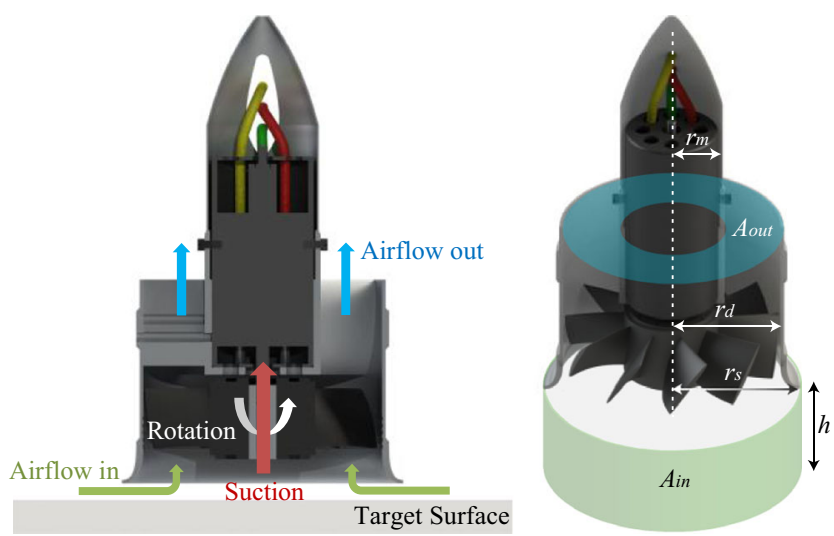

Fig. 3 Vortex adhesion operating principle as shown from the halfsection view of an EDF, with highlighted geometrical parameters
In addition to the adhesion force produced through the negative pressure, the EDF will generate a thrust as it pulls air through the duct. At small $h$, the air density will not be enough to create a big thrust force, but as the gap increases and airflow is less restricted, the generated thrust will increase. Ultimately the thrust generated will converge to its free flight equivalent [17].

The structural design of the duct has an impact on the fluid velocity according to Bernoulli's theory [15]. Bernoulli's theory states that when a fluids velocity along a stream line increases, the pressure along the stream line drops. This means that when the rotational speed of the EDF increases and the air moves faster through the shroud, an additional pressure drop will be induced.

\section{VAS Setup Design and Prototype Components}

The proposed experimental methodology will provide a detailed overview of the complicated pressure/force phenomena taking place while the VAS operates, while extracting the acquired sensorial data will help investigate their connection to the related theoretical background. Ultimately, these derived theoretical connections between the fundamental properties (pressure, force, power consumption) will lead to better descriptions, models and control algorithms for this system.

\subsection{Conceptual Design}

The VAS experimental setup was designed with the main goal of measuring the adhesion force $F$ exerted from a test EDF to the test surface. To achieve this, as represented in Fig. 4, the EDF is mounted to a legged support structure where each of the four legs are connected to the baseplate via a load cell, resembling the rectangular formation of commercial weight scales. In this configuration, the total measured force $F$ is derived by the summation of the force measured by the four load cells, displayed in Fig. 4 as $F_{\{1, \ldots, 4\}}$.

One of the main design goals for the VAS was to be easily adaptable for fitting EDFs of different dimensions and design characteristics. As shown in Fig. 4a and b, the VAS structure is properly modified to incorporate the two typical EDF designs presented in Fig. 1a and b, respectively.

Another design goal of the presented setup was the measurement of the pressure alterations during the VAS's operation, for which the sensing areas of interest had to be properly defined. Measurements on the test surface will provide the information needed to investigate the generated negative pressure, its distribution along the test surface underneath the blades, shroud or away from the EDF and 
Fig. 4 Graphical representation of the VAS experimental setup for the two EDF designs presented in Fig. 1: (top) side view, (middle) bottom semi-transparent view with highlighted sensor components, (bottom) half-section view with highlighted design variables
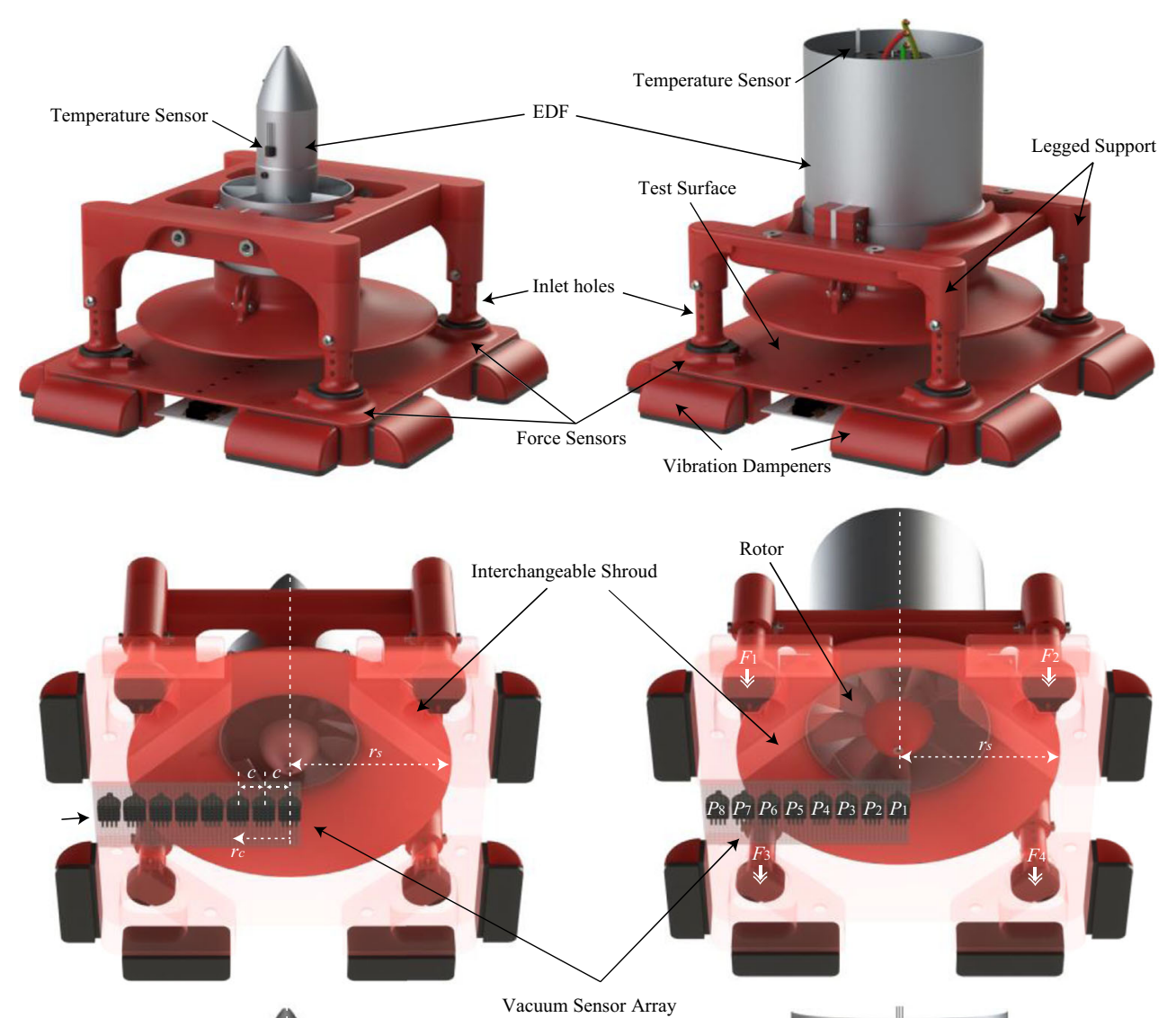

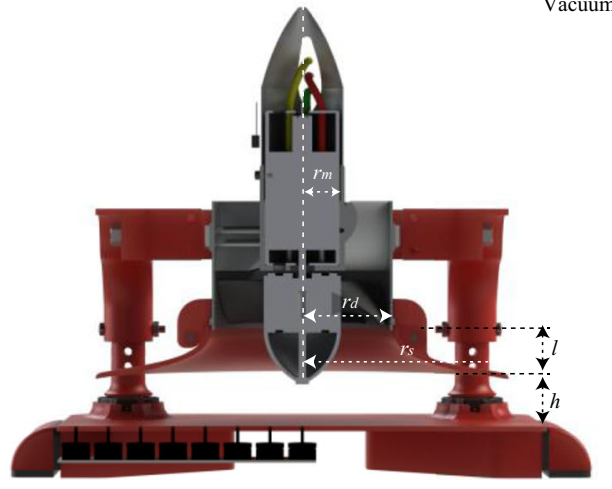

(a)

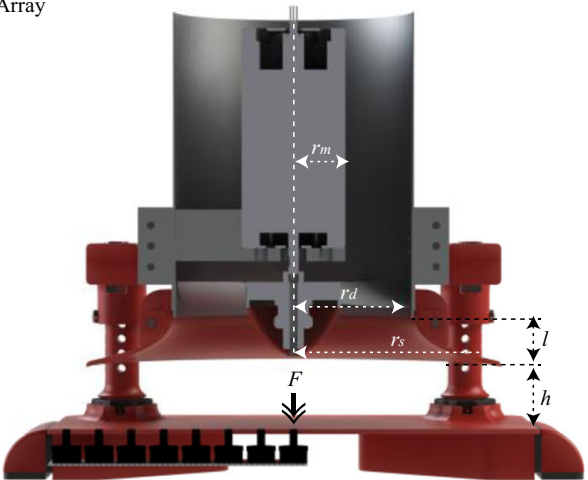

(b) give an insight on its physical nature. In the conceptual Computer-Aided-Design (CAD) presented in Fig. 4, a vacuum sensor array was placed underneath the test surface with the sensing tips placed coincidently through inlet holes of the surface at equal intervals $c$ and distance $r_{c}$ from the center point.

Provided the general symmetry of the VAS structure, the flow distribution along circular sections around the test EDF's longitudinal axis in its interior and exterior space was assumed to be symmetrical, while the aerodynamic effect of the legged structure was assumed negligible. For these reasons, the sensor array was placed radially from the surface center, which is defined as the intersection of the test EDF's longitudinal axis and the target surface. In this way, the setup provides with a series of pressure sensor points, denoted as $P_{\{1, \ldots, 8\}}$.

To expand the evaluation span of the VAS adhesion properties, different design variables in the setup and modifications to the original EDF structure were incorporated. Specifically, the distance of the test EDF from the test surface $h$ was conceptualized as variable and to this purpose the legged structure was equipped with multiple, inlets placed at predetermined intervals (as shown in Fig. 4a and b). The legged parts were properly adjusted in height to have the 
shroud touching the surface when connected to the bottom inlet $(h=0)$. In addition, shrouds of different outer radius $r_{s}$ were designed to be interchangeable via mounting brackets for evaluating the effect of the active surface and volume change on the attraction between the VAS and the test surface for a given distance. For the needs of this study, the design of the shrouds followed the outward curvature profile utilized in commercially available EDFs, while their height $l$ was selected as constant and predefined.

An additional design criterion was the incorporation of a temperature sensor on the VAS motor cover. These temperature measurements could indicate a possible overheating in cases where the motor does not get sufficiently cooled by the generated airflow. Finally, to increase the safety of operating the VAS at high throttle values and block the translational motion of the setup, mainly due to vibrations from the mechanical parts, vibration dampeners were incorporated symmetrically around the surface to prevent such undesired movements.

\subsection{VAS Prototype Components}

To expand the experimental evaluation of the VAS concept, the design was properly adjusted for the prototype to incorporate two commercially available EDF models resembling the designs displayed in Fig. 4. Specifically, for the needs of the presented study, two EDFs developed by Hobbyking (from here on referred as models A and B) were utilized, with their corresponding specifications presented in Table 1. The presented models were selected for their different design parameters and motor specifications, while both being characterized by high thrust-to-weight ratios (7.83 and 5.67 as calculated from Table 1, respectively); a vital characteristic for their potential use in a WCR.

The VAS prototype, which is displayed in Fig. 5 for both test-EDF cases, was 3D printed from Polylactide (PLA) in Ultimaker $2+$ printers following the required design properties for the execution of this study, while the dimensions were adjusted to the needs of the selected test

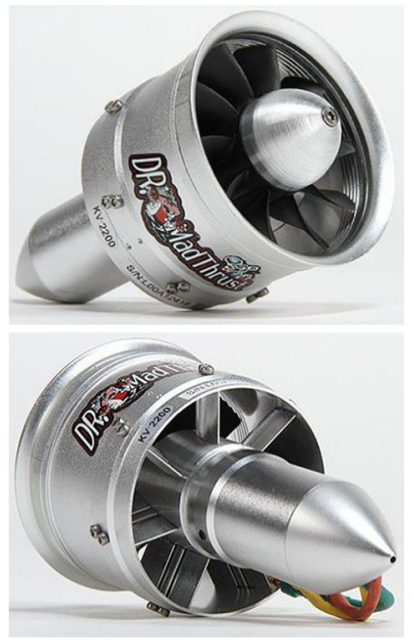

(A)

Material
Duct Diameter (mm)
Duct Thickness (mm)
Weight $(\mathrm{kg})$
Rotor Fan
Motor Type
Max RPM (rpm)
Max Power (W)
Continuous Power (W)
Max Voltage (V)
Max Current (A)
Max Thrust (kg)

Material

Duct Diameter (mm)

Duct Thickness (mm)

Motor Type

Max RPM (rpm)
Aluminium Alloy

70

0.75

0.31

10 Blade Fan

Brushless DC 2200kV

48000

1900

1700

22.2

76

2.45

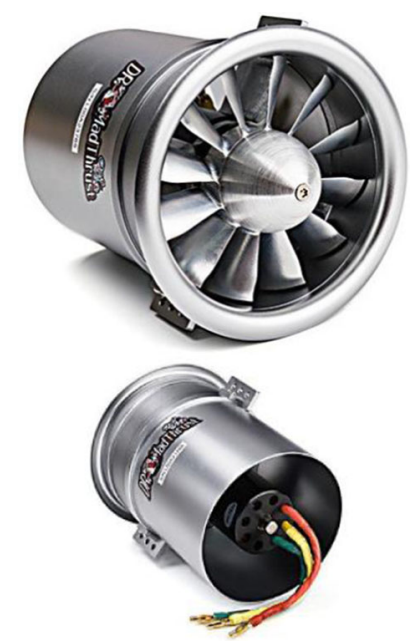

(B)

Aluminium Alloy

92

0.75

0.67

12 Blade Fan

Brushless DC $1600 \mathrm{kV}$

41000

2350

2200

22.2

98

3.80 
Fig. 5 VAS prototype for the two EDF cases with highlighted sensor and data acquisition components
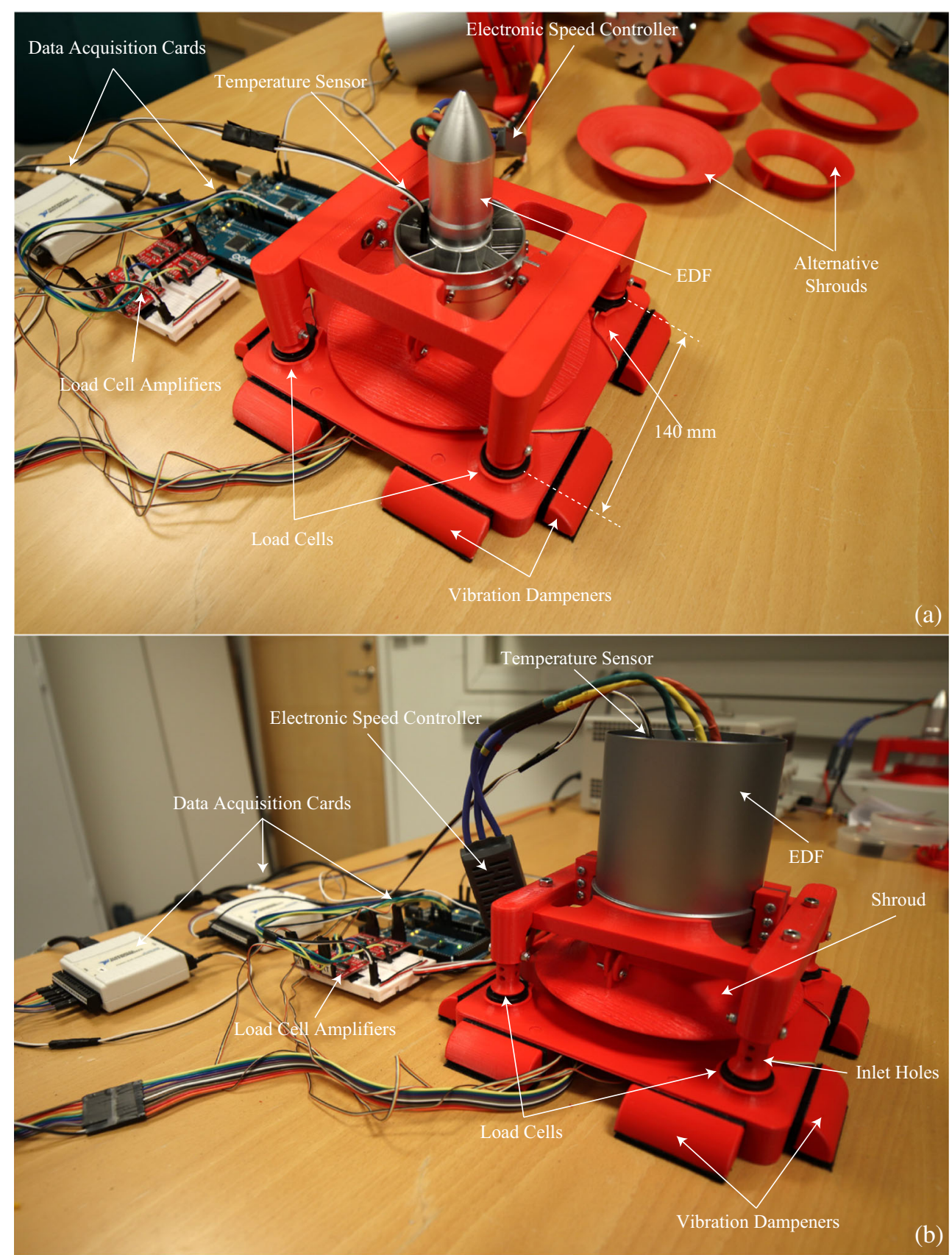

EDF and the utilized sensors. Specifically, the test surface was printed as a $190 \times 190 \times 2 \mathrm{~mm}$ (Length $\times$ Width $\times$ Height) plate, with incorporated inlet holes for the pressure sensors, as well as mount extrusions for the load cells (Fig. 6). In this prototype setup, the gap height $h$ can be set between 0 and $21 \mathrm{~mm}$ in $3 \mathrm{~mm}$ increments. This is achieved via the inlet holes of the structure's four legs, placed in a $140 \times 140 \mathrm{~mm}$ formation for uniformal force distribution (Fig. 5).

The pre-built aluminium shroud in both test cases was replaced by four different renditions, which were 3D printed with outer radius $r_{s}=50,60,70$ and $80 \mathrm{~mm}$, following an outward curvature profile and constant shroud thickness $2 \mathrm{~mm}$, while all the shroud heights were predefined at $l=$ $22 \mathrm{~mm}$.

For measuring the force $F$ generated from the EDF and acting on the test surface via the legged structure, four TE Connectivity FX1901 button-type load cells, with $11.34 \mathrm{~kg}$ maximum range, were utilized and properly incorporated in between the test-surface and respective leg tips (Fig. 6(right)), to ensure uniformal tension on the active button element of each force sensor and thus more reliable 
Fig. 6 Details of the VAS prototype: (left) bottom view of the test EDFs, (right) side view of the test surface

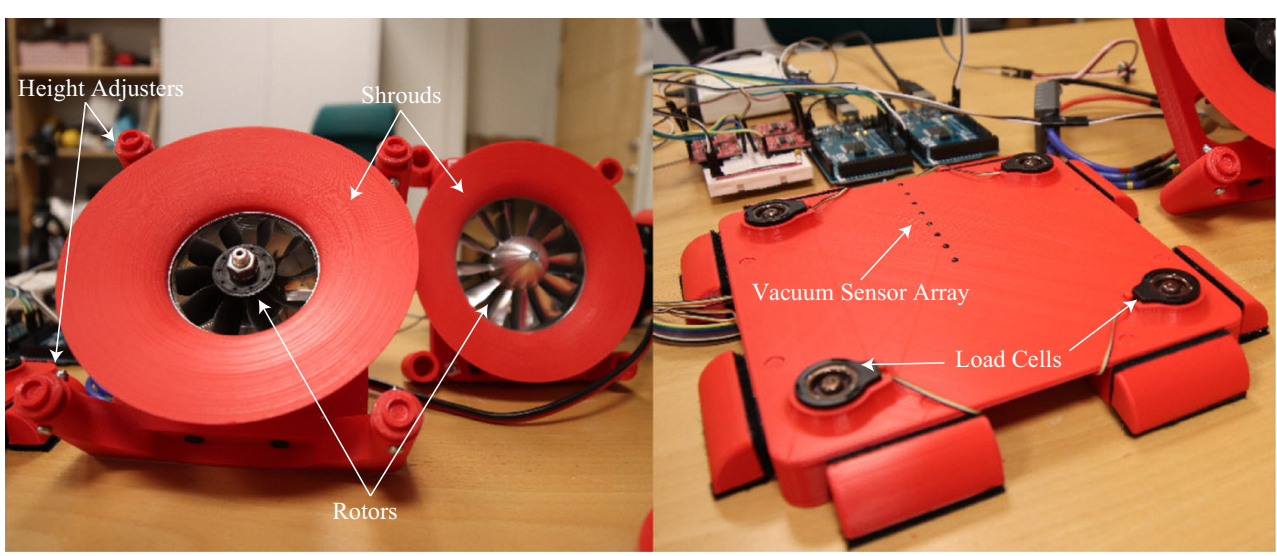

measurements. The load cell amplifiers selected for these experimental trials were four SparkFun HX711.

The Electronic Speed Controller (ESC) selected for both tested EDF units was the Turnigy AE-100A with continuous current capability of $100 \mathrm{~A}$ and characterized by a fast and precise throttle response. To handle the high current requirements of the selected EDFs, a EA-PSI 8080-70 2U power supply was selected, with the ability of providing high output voltages $(0-720 \mathrm{~V})$ and currents $(0-120 \mathrm{~A})$.

The acquisition of the negative pressure measurements was achieved via multiple NXP USA Inc. MPXV5050V differential pressure sensors with a sensing range of up to $-50 \mathrm{kPa}$. More specifically, an array of eight sensors was placed in a single radial formation along the test surface; starting from the surface center, i.e. the intersection of the test surface with the EDF's longitudinal axis. The sensors were placed radially at a $c=12.5 \mathrm{~mm}$ interval that was dictated by the sensor dimensions. Due to the differential principle of their operation, the tip sensing point targets the test surface (Fig. 6), while their capsule reference point remains in the opposite side of the surface and thus unaffected by the generated airflow.

An Analog TMP37 temperature sensor was properly mounted on the motor case to monitor its thermal levels during operation. These measurements were ultimately utilized to ensure the setup's safe operation during the experimental sequences. To further increase the safety of operating the VAS at maximum throttle and block the translational motion of the setup during those extreme conditions, custom vibration dampeners were finally incorporated around the test surface to adequately prevent such undesired movements.

Finally, the acquisition of the setup's sensorial data and the control of the EDF's operation was achieved via two National Instruments USB-6008 cards and two Arduino Mega boards, while the main control and programming of the setup was performed in a personal computer system (Windows 7, 64-b, Intel i7 processor, 32-Gb RAM).

\section{Experimental Evaluation of the VAS Adhesion Properties}

This section deals with the need to evaluate the VAS adhesion properties in an effort to define and adjust its design characteristics for its future incorporation to a WCR of set requirements and specifications.

\subsection{Shroud and Active Inlet Area Alterations}

Initially, for the experimental evaluation of the VAS properties under the two selected EDF cases, multiple trials were performed for different combinations of shroud radius $r_{s}$ and gap height $h$ values. The experimental cases presented in the sequel were performed with the goal to:

a) find the design variables that provides this EDF setup's optimal performance,

b) analyse the nature of the pressure and force alterations related to the throttle input,

c) investigate whether the vortex is the evident and sole factor for generating adhesion in all shroud and gap height cases.

For the needs of the presented experimental sequences, the program sampling frequency was set at $40 \mathrm{~Hz}$, while the throttle $T$ input signal was selected as a pyramid function of $5 \%$ increments every $2 \mathrm{~s}$, with a peak throttle $T_{\max }=100 \%$ and then reversed down in the same manner until reaching $T_{\min }=0 \%$.

Force measurements were acquired as the summation of the four load cells $F=\sum F_{l}, l=1, \ldots, 4$, while subtracting the weight of the VAS acting on the force measurement points to properly calibrate the adhesion force measurement.

The points $P_{n}, n=1, \ldots, 8$, denote the vacuum sensors radially and outwards from the surface center, starting with $P_{1}$ as the center sensing point. It is important to note that for the variable shroud radii $r_{s}$, the sensors lying under 
the VAS and shroud differ. Specifically, for $r_{s}=50,60$, 70 and $80 \mathrm{~mm}$ the sensors $P_{\{1, \ldots, 4\}}, P_{\{1, \ldots, 5\}}, P_{\{1, \ldots, 6\}}$ and $P_{\{1, \ldots, 7\}}$ lie within the outer radius, respectively, while in all aforementioned cases, $P_{\{1, \ldots, 3\}}$ and $P_{\{1, \ldots, 4\}}$ are positioned under the (A) and (B) EDF duct, respectively. The vacuum sensor array was properly placed so that there are always sensors placed outside the affected surface area, to provide an insight on how the pressure is affected in these cases.

Prior and after the performed experimental sequences, all sensors were properly calibrated, and every sequence was repeated several times to ensure the repeatability and conformity of the presented results.

An indicative experimental test was executed for the two EDF cases using $r_{s}=70 \mathrm{~mm}$ and (A) $h=3 \mathrm{~mm}$, (B) $h=$ $6 \mathrm{~mm}$, where the acquired throttle input $T$, negative pressure $P n$, force $F$ and power consumption $P_{c}$ are presented in Fig. 7.

The experimental tests showed that the increase in the throttle causes a clear increase in the negative pressure for both cases, which becomes progressively greater for the pressure sensors closer to the surface center; and thus revealing the generation of a vortex. The maximal negative pressures were recorded at $T=100 \%$ and reached approximately (A) $5.8 \mathrm{kPa}$ and (B) $6.5 \mathrm{kPa}$ for the sensor $P_{1}$.
The neighboring sensors provided similar measurements but their larger range profile appearing as noise, which reveals the different effect from the vortex generation. In both cases, the sensor $P 8$ that is positioned outside of the affected area of the chosen shroud, measured a smaller pressure change as the air volume is bigger and the inlet area restricted, but the entirety of the low-pressure zone was thus not confined to just the volume between the shroud and the surface, but extended further and outside the enclosed volume. Case (A) was affected more by vibration phenomena and observed resonant points, which are evident on the higher amplitude alterations in pressure distribution measurements in comparison to case (B).

In addition, the measured force $F$ showed a behavior proportional to the throttle, following the quick response characteristics of the negative pressure signals. As expected, maximum force was acquired for maximum throttle and was measured at (A) $28 \mathrm{~N}$ and (B) $23 \mathrm{~N}$. In overall, the presented experimental trials provided a clear indication of the generated negative pressure vortex and the resulting adhesion, as already recorded in related literature [3]. In more detail, the presence of transient phenomena was evident of the ESC behavior adjusting the power consumption and become more intense in case (B) for
Fig. 7 Indicative experimentally acquired responses, displaying negative pressure $P_{n}$, total force $F$ and power consumption $P_{c}$ of the two EDF cases $(\mathbf{a}-\mathbf{b})$, for the same input throttle signal $T$ and the noted cases of shroud radius $r_{s}$ and gap $h$
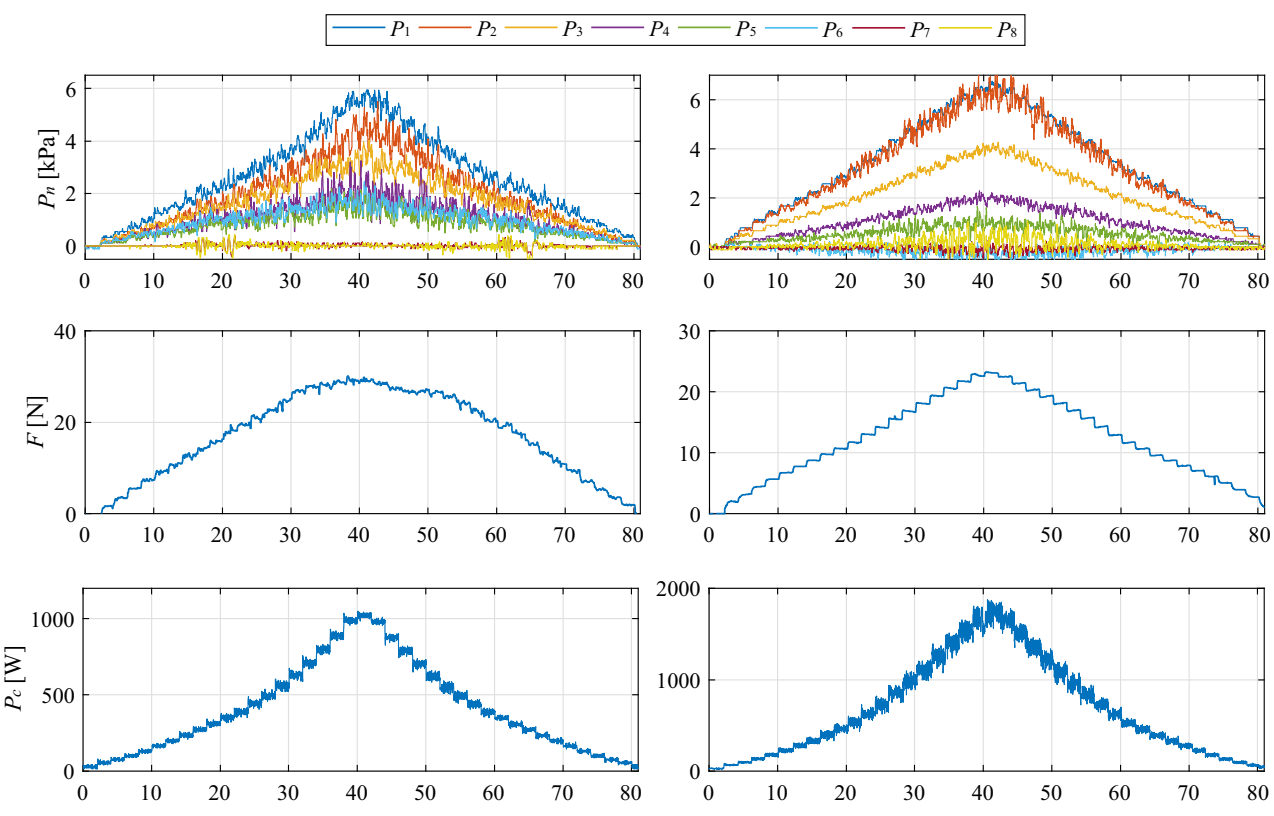

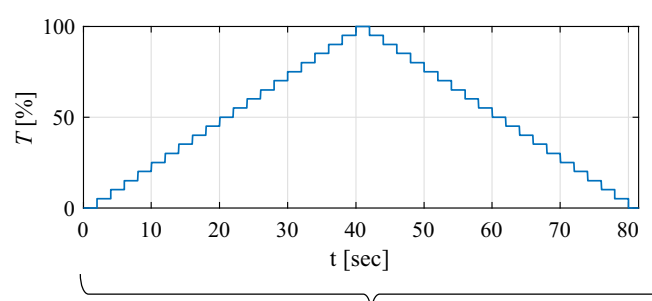

(a) $r_{s}=70 \mathrm{~mm}, h=3 \mathrm{~mm}$

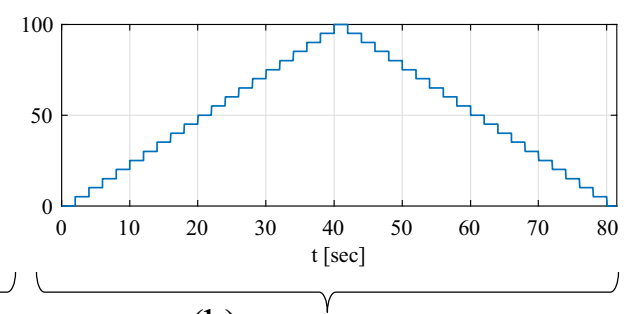

(b) $r_{s}=70 \mathrm{~mm}, h=6 \mathrm{~mm}$ 
higher throttle. As mentioned, case (A) was generally susceptible to higher vibration and intense resonant points that, in the presented experiment, had a direct effect on the quality of the force responses which degraded when reaching higher throttle values.

It must be noted that all acquired VAS open-loop responses for the specified input command were characterized by a short time constant of approximately $0.2 \mathrm{~s}$, which reached the maximum $0.4 \mathrm{~s}$ for the case of single-step throttle change from 0 to $100 \%$ and is directly affected by the ESC performance as well as the EDF physical response characteristics. A delay of $0.2 \mathrm{~s}$ was also observed between the throttle change and the recorded response, which was a direct result of the communication delays between the computer system, the DAQ card and the ESC. The overall response of the VAS was also directly affected by factors as the power wire characteristics and the ESC temperature. For these experiments, a 2-meter $12 \mathrm{AWG}$ wire was utilized, while the length increase led to an expected drop on the ESC achieved current draw from the power supply. In extreme cases where the ESC temperature reached critical levels after long-hour experimentation, a drop in performance of about $10 \%$ was identified, which makes the ESC monitoring and cooling an important factor for future iterations of the
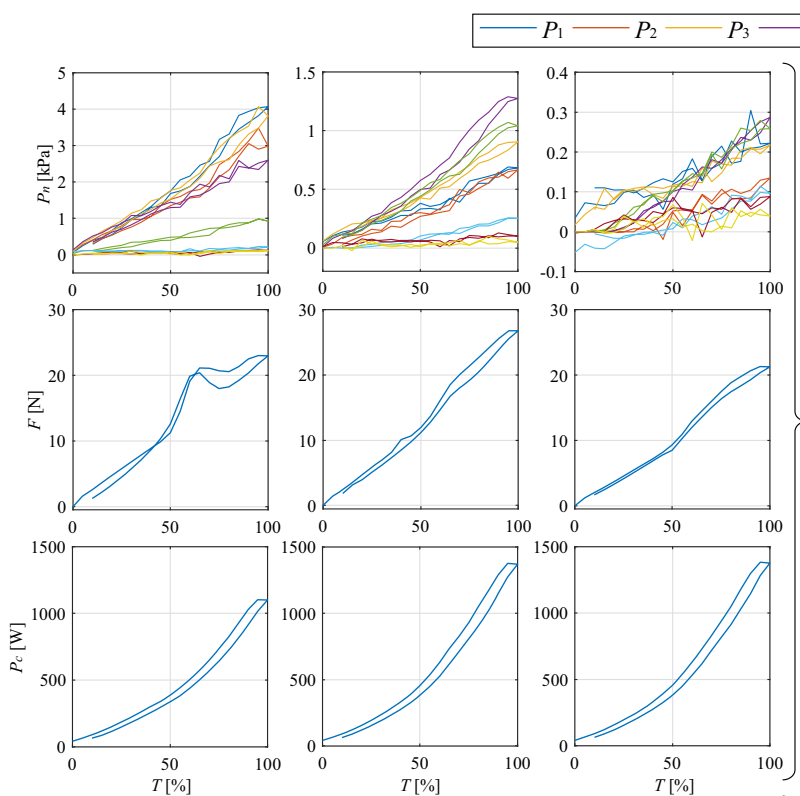

(a)
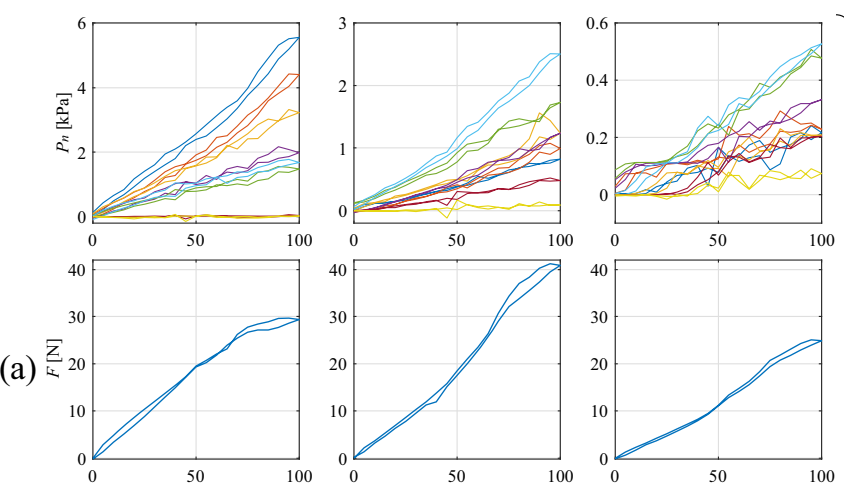

(c)
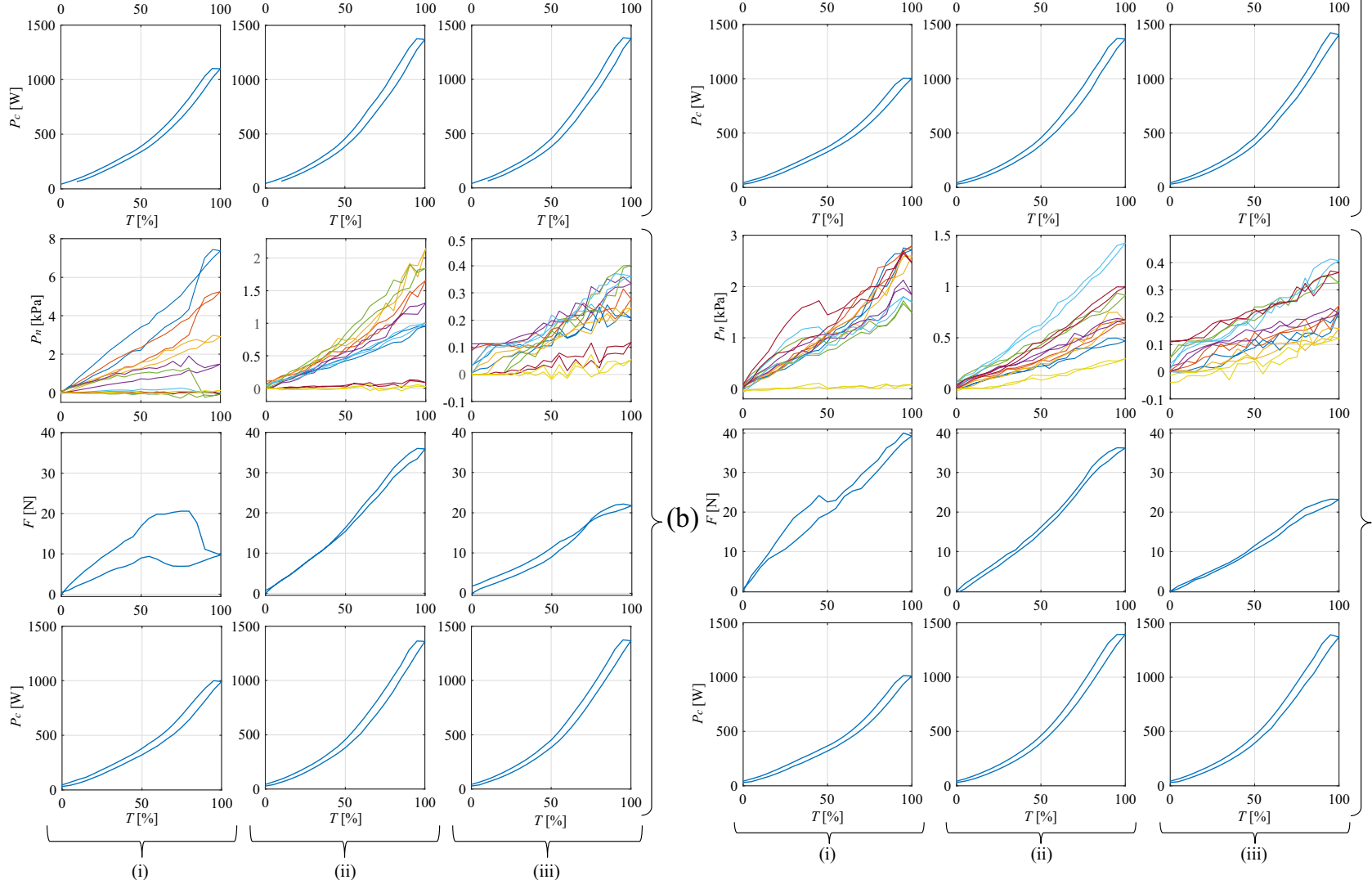

Fig. 8 Negative pressure $P_{n}$, force $F$ and power consumption $P_{c}$ hysteretic relations to the throttle input signal for EDF (A), gaps $h=$ (i) 3 , (ii) 9, (iii), $18 \mathrm{~mm}$ and shroud radii $r_{s}=\mathbf{a} 50, \mathbf{b} 60, \mathbf{c} 70, \mathbf{d} 80 \mathrm{~mm}$ 
VAS. All above characteristics have been verified for their repeatability through multiple experimental sequences.

To the goal of further investigating the pressure and force relation to the throttle input, the aggregated $P_{n}, F$ and $P_{c}$ responses for $h=3,9,18 \mathrm{~mm}$ and $r_{s}=50$ $80 \mathrm{~mm}$ in $10 \mathrm{~mm}$ increments were acquired for the same throttle input signal presented in Fig. 7. The presented relations extracted from these experiments are displayed in Fig. 8 for case (A) and in Fig. 9 for case (B). For both EDFs and all presented cases of different gaps and shrouds all measured properties follow a hysteretic nature to the changes in throttle, with the upper and lower branches of the hysteresis loops progressing with throttle increase and decrease respectively.

Furthermore, from the obtained experimental results, it can be observed that all maximum measurements of negative pressure, force and power were acquired at maximum $100 \%$ throttle. For both EDF cases and for the same gap $h$, an increase in the shroud radius $r_{s}$ leads to a progressive increase in all maximum values, a phenomenon that can be explained by the increase in the active shroud area $A$ as defined in Section 2.

An important observation is that although an increase in $h$, for the same $r_{s}$, leads to a significant decrease in the $P_{n}$
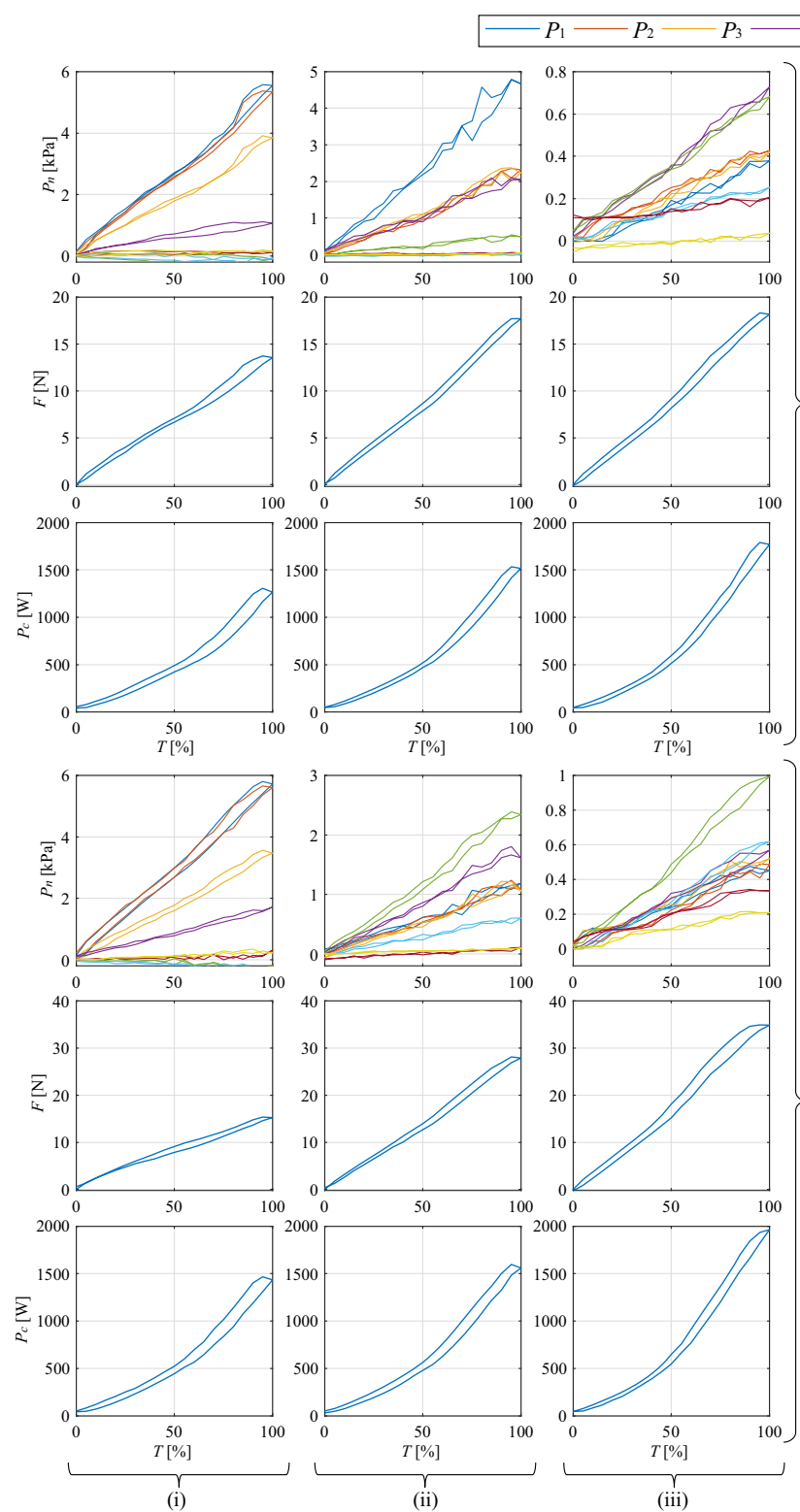

(a)
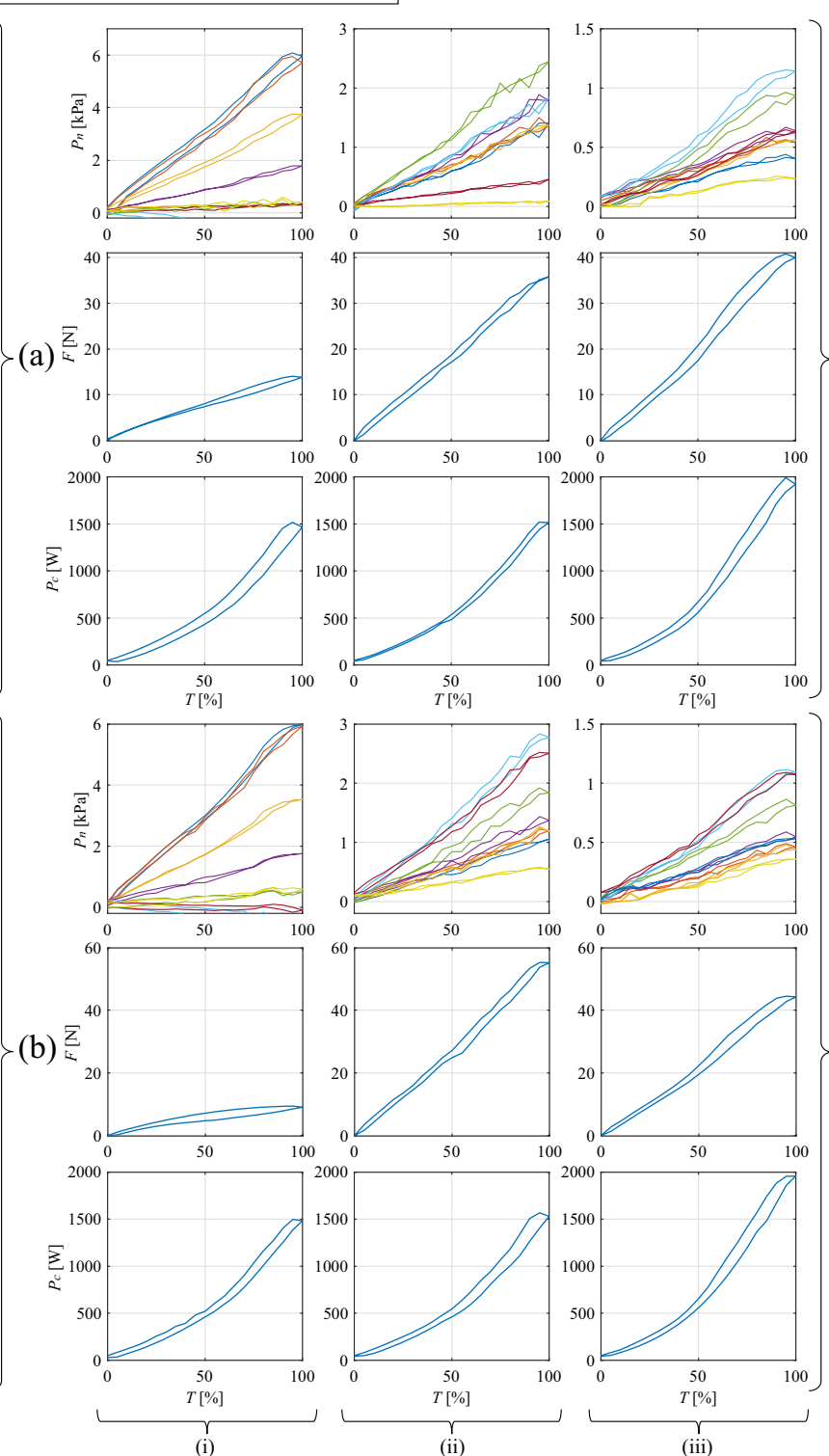

(d)

Fig. 9 Negative pressure $P n$, force $F$ and power consumption $P_{c}$ hysteretic relations to the throttle input signal for EDF (B), gaps $h=$ (i) 3, (ii) 9, (iii), $18 \mathrm{~mm}$ and shroud radii $r_{s}=\mathbf{a} 50, \mathbf{b} 60, \mathbf{c} 70, \mathbf{d} 80 \mathrm{~mm}$ 

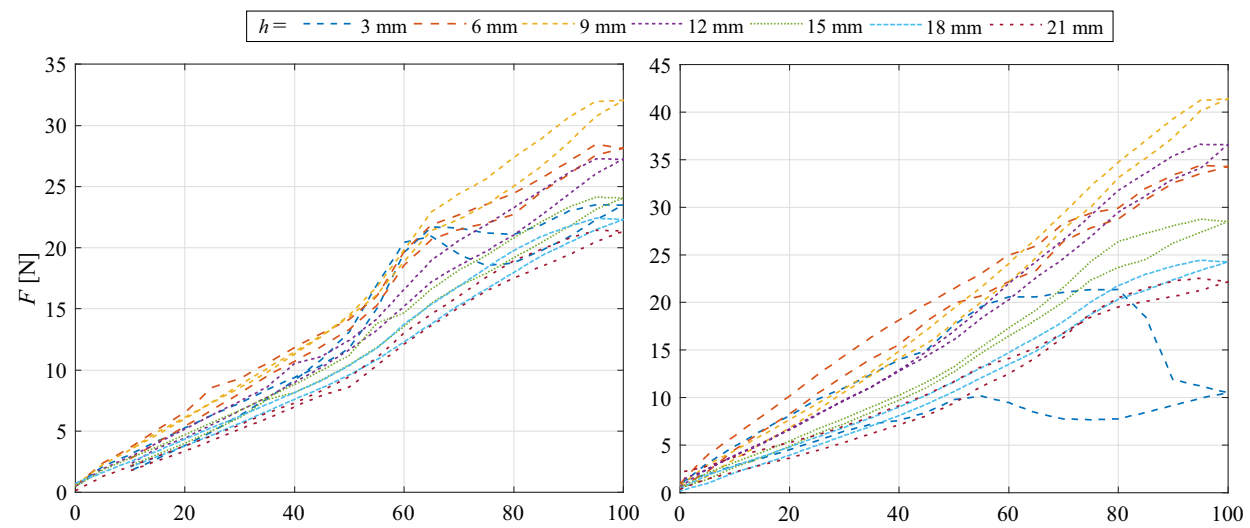

(a)

(b)
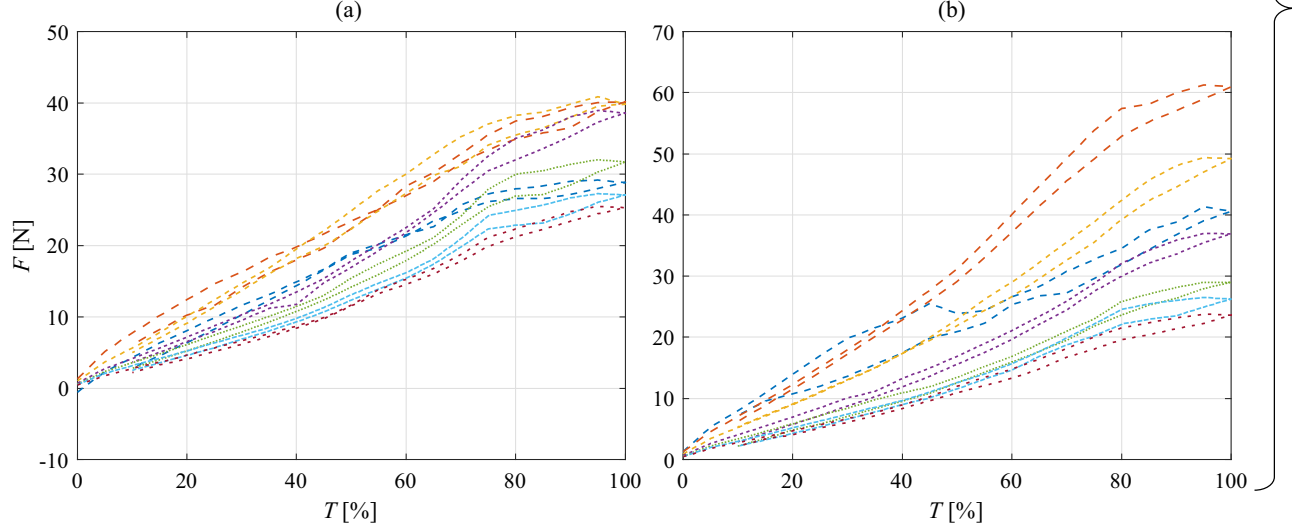

(a)

(c)

(d)

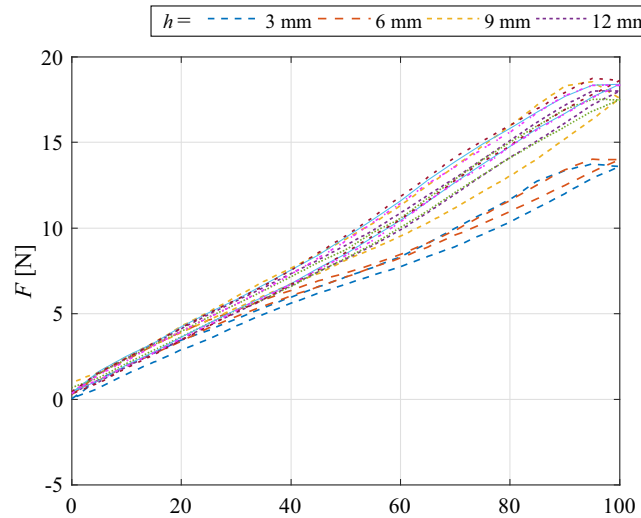

(a)

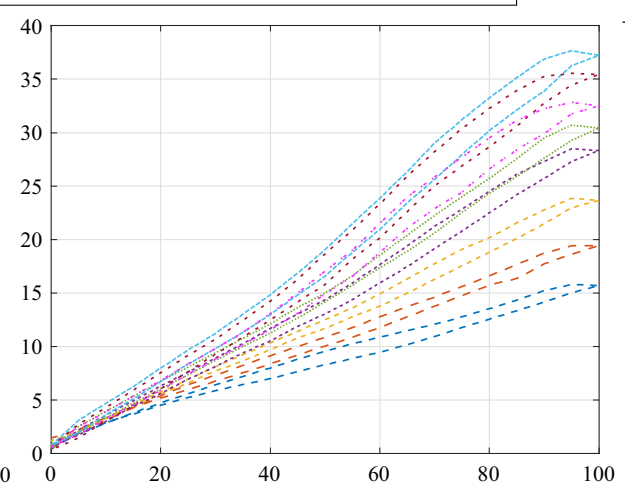

(b)
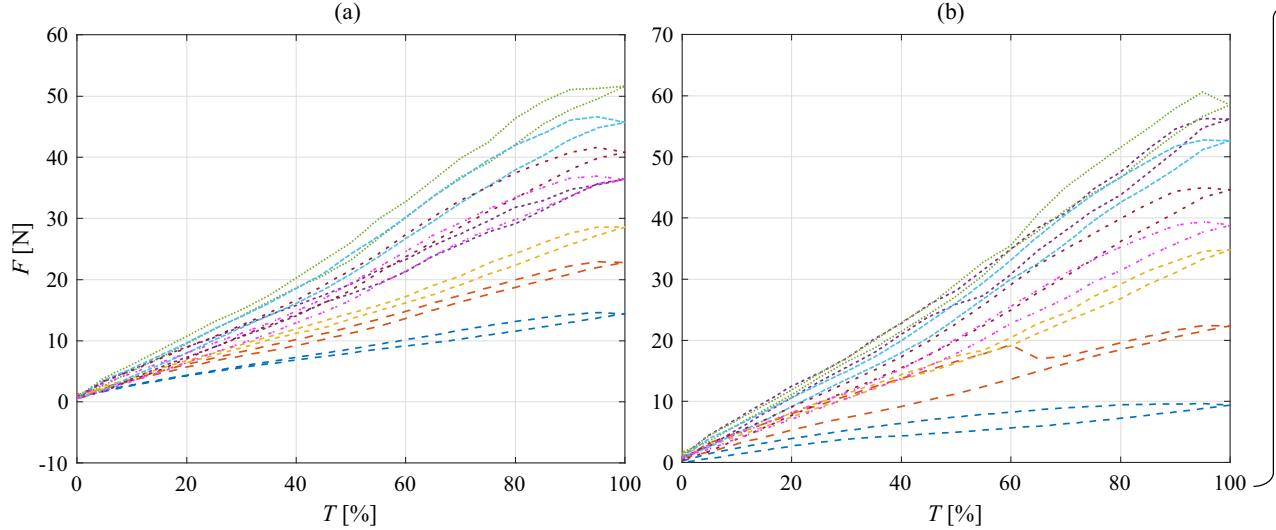

(b)

(c)

(d)

Fig. 10 Transition of force $F$ to throttle $T$ hysteresis loops for both EDF cases (A-B), gaps $h=3-21 \mathrm{~mm}$ and shroud radii $r_{s}=\mathbf{a} 50, \mathbf{b} 60, \mathbf{c} 70$ and $\mathbf{d} 80 \mathrm{~mm}$ 
Table 2 Maximum adhesion forces and respective gaps for cases (A-B) and different shroud radii

\begin{tabular}{|c|c|c|c|c|}
\hline & $r_{s}=50 \mathrm{~mm}$ & $r_{s}=60 \mathrm{~mm}$ & $r_{s}=70 \mathrm{~mm}$ & $r_{s}=80 \mathrm{~mm}$ \\
\hline (A) & $32.1 \mathrm{~N} @ h=9 \mathrm{~mm}$ & $40.7 \mathrm{~N} @ h=9 \mathrm{~mm}$ & $46.6 \mathrm{~N} @ h=9 \mathrm{~mm}$ & $61.1 \mathrm{~N} @ h=6 \mathrm{~mm}$ \\
\hline (B) & $18.1 \mathrm{~N} @ h=21 \mathrm{~mm}$ & $37.2 \mathrm{~N} @ h=18 \mathrm{~mm}$ & $51.2 \mathrm{~N} @ h=15 \mathrm{~mm}$ & $60.2 \mathrm{~N} @ h=15 \mathrm{~mm}$ \\
\hline
\end{tabular}

measurements, the adhesion force does not follow the same decreasing behaviour.

To better illustrate that phenomenon, the transition of force $F$ to throttle $T$ hysteresis loops for both EDF cases (A-B), gaps $h=3-21 \mathrm{~mm}$ in $3 \mathrm{~mm}$ increments and shroud radii $r_{s}=50-80 \mathrm{~mm}$ in $10 \mathrm{~mm}$ increments are presented in Fig. 10. In most cases, the hysteretic nature of this system ranges from $4-6 \%$, while reaching peaks of $20 \%$ in cases of $h=3 \mathrm{~mm}$. Knowledge of this phenomenon will play an important role in the future modeling and control of the VAS, as this data will be utilized for its identification and compensation.

For all shroud radii, the maximum adhesion force appears to increase while $h$ increases, until it reaches a maximum point after which it starts decreasing. The maximum forces for each case were extracted and shown in Table 2, which also highlights the gap value at which that optimum adhesion efficiency is observed.

A comparison between the adhesion efficiency of both EDFs shows that case (A) reaches maximum adhesion forces at smaller $h$ than case (B), while those optimum distances decrease with the increase of $r_{s}$ in both cases. Case (A) is also characterized by larger maximum forces for smaller $r_{s}$ values $(50,60 \mathrm{~mm})$, while for $r_{s}=70 \mathrm{~mm}$ case (B) presents increased efficiency. The maximal forces appear for both EDFs on the largest shroud $\left(r_{s}=80 \mathrm{~mm}\right)$ and their values are comparable and close to $60 \mathrm{~N}$.

The above observations are also visible in Fig. 11, which displays the maximum mean force measurements extracted for the test EDFs on all four shrouds and available gaps. Specifically, an increase in $r s$ leads to an increase in maximum mean forces, which agrees with (1), given the increase in the active area $A$. For case (A) and for each $r_{s}=$ $50,60,70$ and $80 \mathrm{~mm}$, the respective maximum increase in $F$ is translated as approximately $33.8 \%, 69.5 \%, 94.1 \%$ and $154.6 \%$, when compared to the free-flight maximum thrust of $24.0 \mathrm{~N}$ (as extracted from Table 1), while the adhesionto-weight ratio reaches the maximum of 19.9. For case (B) and considering the high free-flight maximum thrust of 37.3 $\mathrm{N}, F$ is showing a decrease of 51.5 and $0.3 \%$ for $r_{s}=50$ and $60 \mathrm{~mm}$, while for $r_{s}=70$ and $80 \mathrm{~mm}$ it changes to a maximum increase of $39.7 \%$ and $61.4 \%$, respectively. In this case, the adhesion-to-weight ratio reaches a maximum of 9.15, which further highlights the efficiency of the proposed methodology in both EDF cases.

It is important to note that in case (A) the force measurements are observed to be the numerically identical for all shrouds in very large gaps, while in case (B) that appears to happen for very small gaps. A future repetition of these experiments for gaps smaller than $3 \mathrm{~mm}$, larger than $24 \mathrm{~mm}$ and in smaller increments would provide further information on whether this numerical identity is occurring in both small and large gaps for both EDF cases, as their adhesion efficiency appears to follow a bell-shaped distribution.

Plotting the acquired maximum adhesion forces in relation to the ratio of the inlet and outlet areas $A_{\text {out }} / A_{\text {in }}=$ $\pi\left(r_{d}^{2}-r_{m}^{2}\right) / 2 \pi h r_{s}$, as defined in Section 3, reveals a correlation for both EDF cases on their optimal operating points. Specifically, as displayed in Fig. 12 for both EDFs, those maximal forces are acquired when $A_{\text {out }} / A_{\text {in }}$ converges to 0.8 . The knowledge of this value is an
Fig. 11 Maximum measured adhesion forces for throttle $T=$ $100 \%$, in relation to the gap height for the two EDF cases (a-b) and the four different shroud radii $r_{s}$

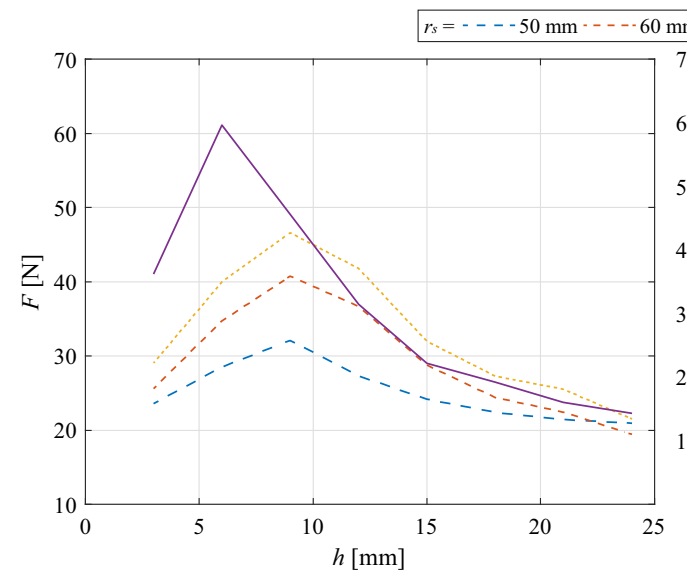

(a)

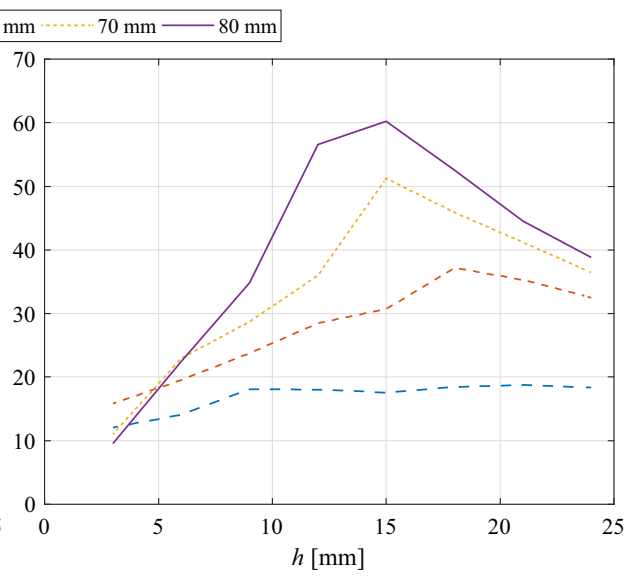

(b) 
Fig. 12 Maximum measured adhesion forces for throttle $T=$ $100 \%$, in relation to the outlet/inlet active area ratio $A_{\text {out }} / A_{\text {in }}$ for the two EDF cases (a-b) and the four different shroud radii $r_{s}$

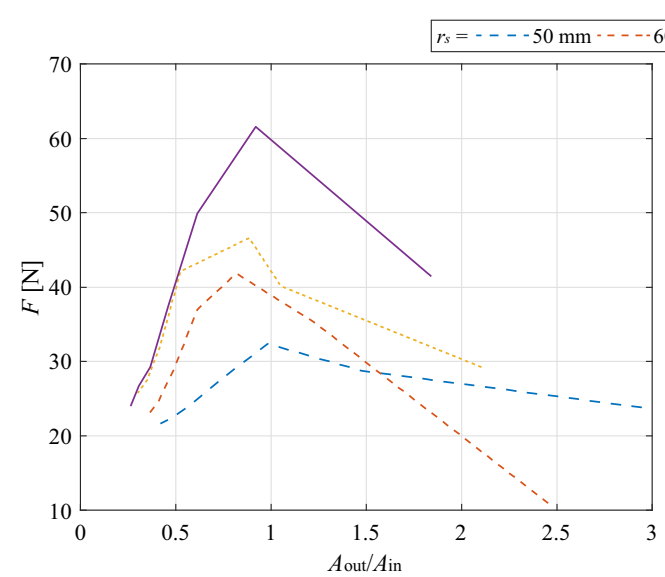

(a)

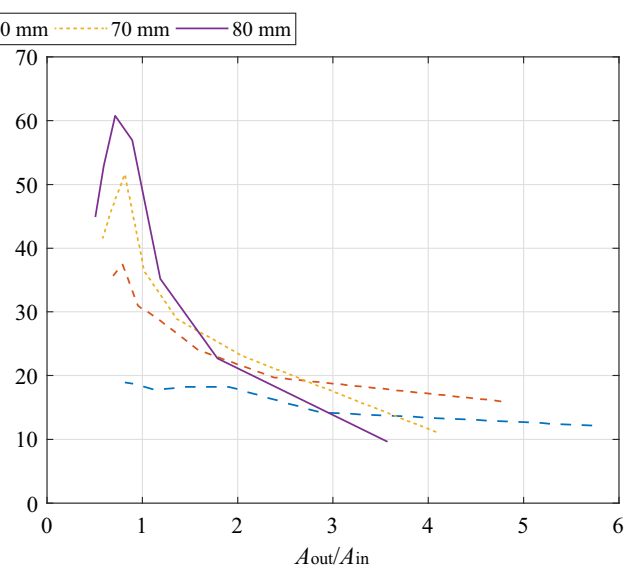

(b) important design tool as it provides the ability to predict the optimal gap $h$ for a given EDF and shroud diameter.

This area ratio convergence to the optimal adhesion point is a highly important finding, since it determines the design details of all future prototypes incorporating the VAS, as trade-offs between EDF dimensions, shroud size and gap are introduced. In an effort to provide an insight to these trade-offs and how they affect the design process according to set specifications and requirements, two cases will be described below, one where the EDF is given and the shroud size is variable, while the second assumes variable EDF dimensions with a constraint in the shroud size.

Given EDF For a given EDF, satisfaction of the ratio $A_{\text {out }} / A_{\text {in }}$ to the optimal point would mean a decrease in the gap $h$ for an increase in $r_{s}$. In other words, the bigger the shroud size, the larger the maximum force that can be achieved but with a smaller gap leading to the optimal behaviour decreases. A very small gap could present a challenge when controlling a WCR, since small variations due to compression of mechanical parts should be taken into consideration to avoid the touching of the shroud to the test surface, which would increase the stress to the VAS and cause a decrease in its performance. A smaller in diameter shroud would mean less maximum force but would give the advantage of having a larger distance from the surface in its optimal performance. This advantage is also translated in system robustness, which is supported by the presented findings, as changes in the distance due to stress leading to compressions of the mechanical components will not significantly affect the performance of the VAS.

Constrained Shroud Size For a given shroud radius $r_{s}$, operating an EDF of smaller $A_{\text {out }}$, which is translated to mean smaller duct radius $r_{d}$ or larger motor radius $r_{m}$, would mean the need for smaller gap to satisfy a constant ratio $A_{\text {out }} / A_{\text {in }}$ and get the optimal performance. In other words, in the design case where the shroud size has a maximum constraint (e.g. to fit in a robotic platform of predefined dimensions), the need for having a system with a specific performance/consumption/weight would affect the EDF dimensions and in turn the optimal gap.

The two design examples are aligned with the presented results (Table 2, Fig. 11), as both cases exhibit their optimal performance for smaller gaps as their shroud radius increases, while the smaller in dimensions case $\mathrm{A}$ maximizes its performance in smaller gaps than case B for a given shroud.

The optimal adhesion force is approximately $60 \mathrm{~N}$ for both cases, thus leading the EDF choice down to the overall performance, power consumption and robustness of the system. Case (A) is consuming less power for the same optimal adhesion and its smaller weight (half of case (B)) indirectly increases the optimal performance due to the larger bandwidth. On the other hand, case (B) provides a performance more robust to changes in the gap, which is important for a robotic system that will have interchangeable modules, thus different weight and different mechanical compressions. Also, the larger duct dimensions and its particular aerodynamic design appears to be less susceptible to mechanical vibrations and resonant frequencies. This choice will be ultimately based to a series of trade-offs and it is a matter that needs further investigation.

The results revealed a change in adhesion nature relevant to the gap that reflects also a complex pressure distribution change along the surface points and is evident for both EDFs. In an effort to further investigate this phenomenon and its underlying causes, the surface pressure distribution $P_{n}$ with respect to the radial distance $r_{c}$ from the surface center, which concede with the pressure sensor points, for all gap heights $h$ and shroud radii $r_{s}$ are presented in Fig. 13.

These results show an increasing behaviour as the shroud radius increases and indicate a clear change in pressure 

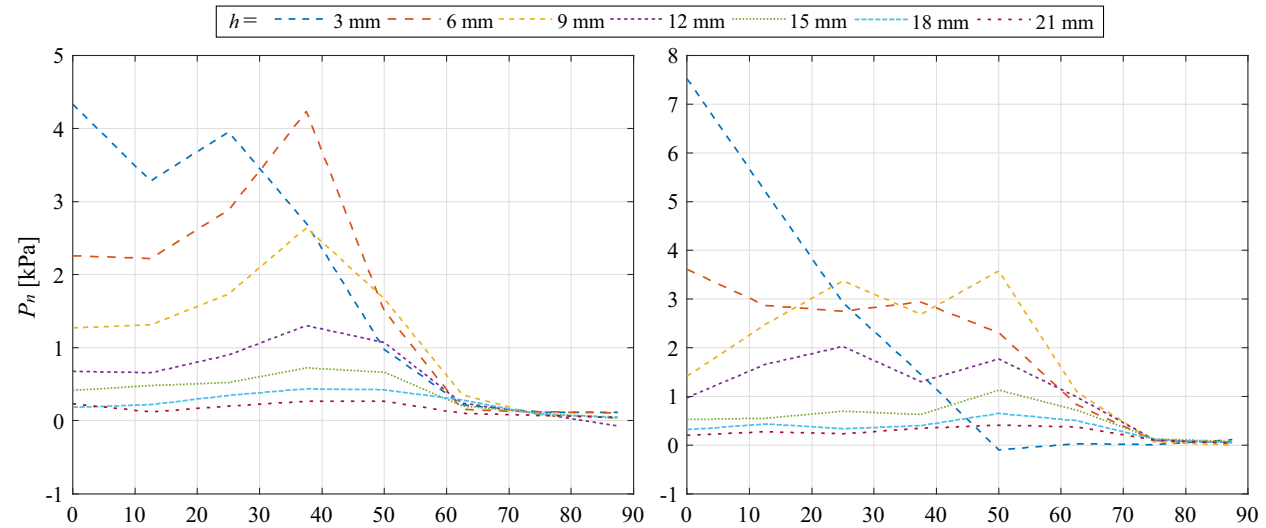

(a)

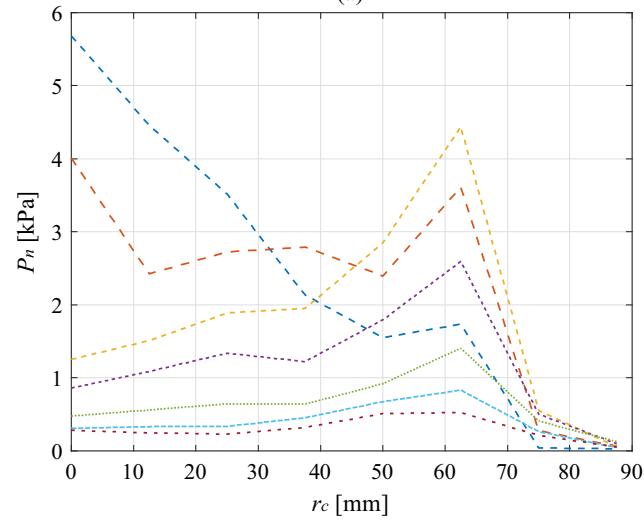

(b)

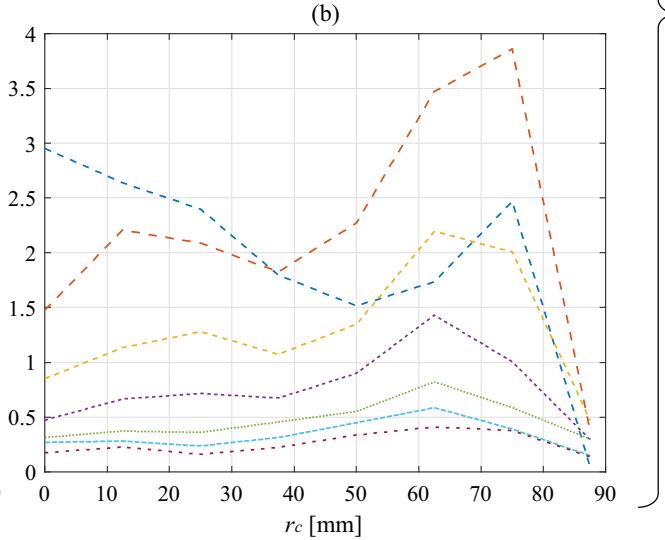

(a)

(c)

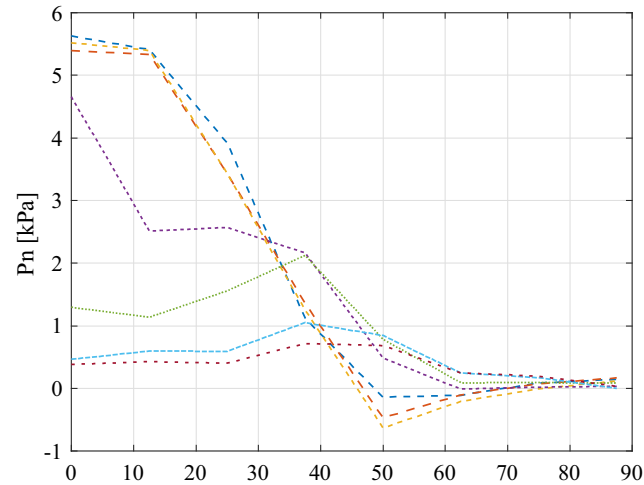

(d)
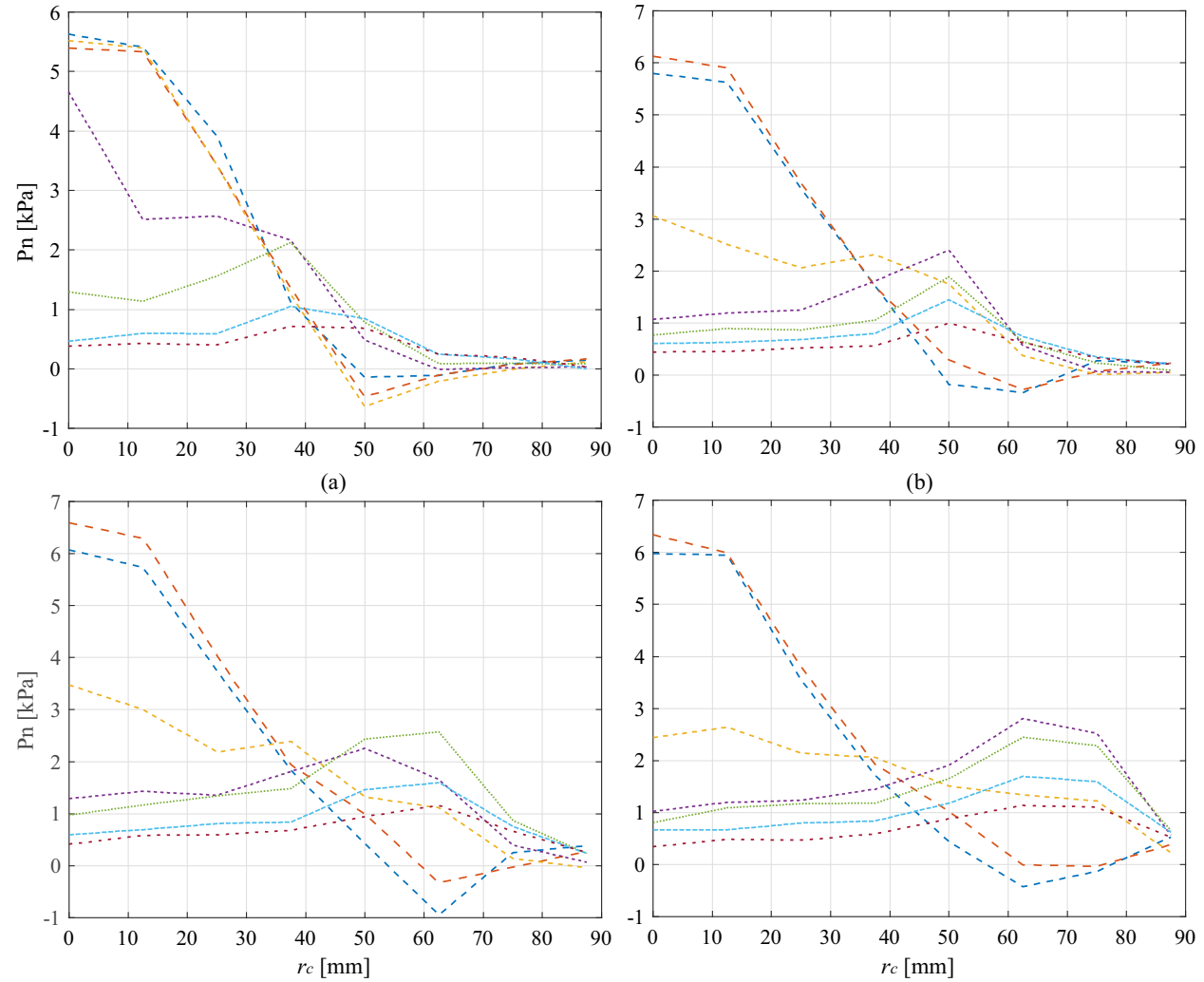

(b)

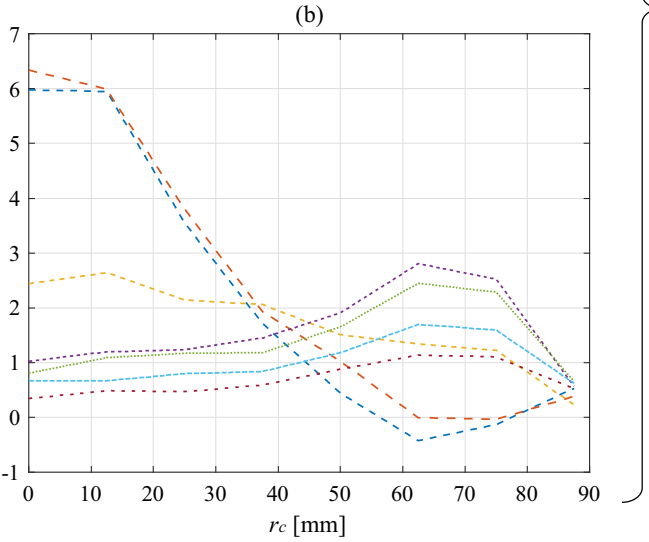

(b)

(c)

(d)

Fig. 13 Measured pressure $P n$ distributions for the two EDF cases with relation to the surface radial distance $r_{c}$ at maximum throttle $T=100 \%$, for gaps $h=3-21 \mathrm{~mm}$ and for shroud radii $r_{s}=\mathbf{a} 50, \mathbf{b} 60, \mathbf{c} 70$ and $\mathbf{d} 80 \mathrm{~mm}$ 
distribution as the gap alters for both EDF cases, with an evident dissipation of the vortex phenomenon and the generation of a ripple-like pressure distribution on the surface points enclosed by the shroud. This change in distribution emphasized by the maximum pressures is rapidly progressing for all shrouds in gaps $h$ between 3$9 \mathrm{~mm}$ for (A) and 6-12 $\mathrm{mm}$ for (B), where the increase in $h$ shifts the dominant pressure points from the nearcenter ones $r_{c}<40 \mathrm{~mm}$ to the middle and outer points of approximately $r_{c}>40 \mathrm{~mm}$.
To better visualize this complex phenomenon and its rapid progression, the experimentally acquired pressure distributions for EDF cases (A-B) is radially expanded to the affected test surface for the shroud case of $r_{s}=80 \mathrm{~mm}$, for $T=100 \%$ and gap heights of $h=3-18 \mathrm{~mm}$ with $3 \mathrm{~mm}$ increments. As displayed in Fig. 14, the pressure values are visualized via different coloring, with an indication of the respective gap $h$ and maximum mean force. As seen for both (A-B) cases, there is a fast progression as the gap increases respectively, where the centralized vortex pressure profile

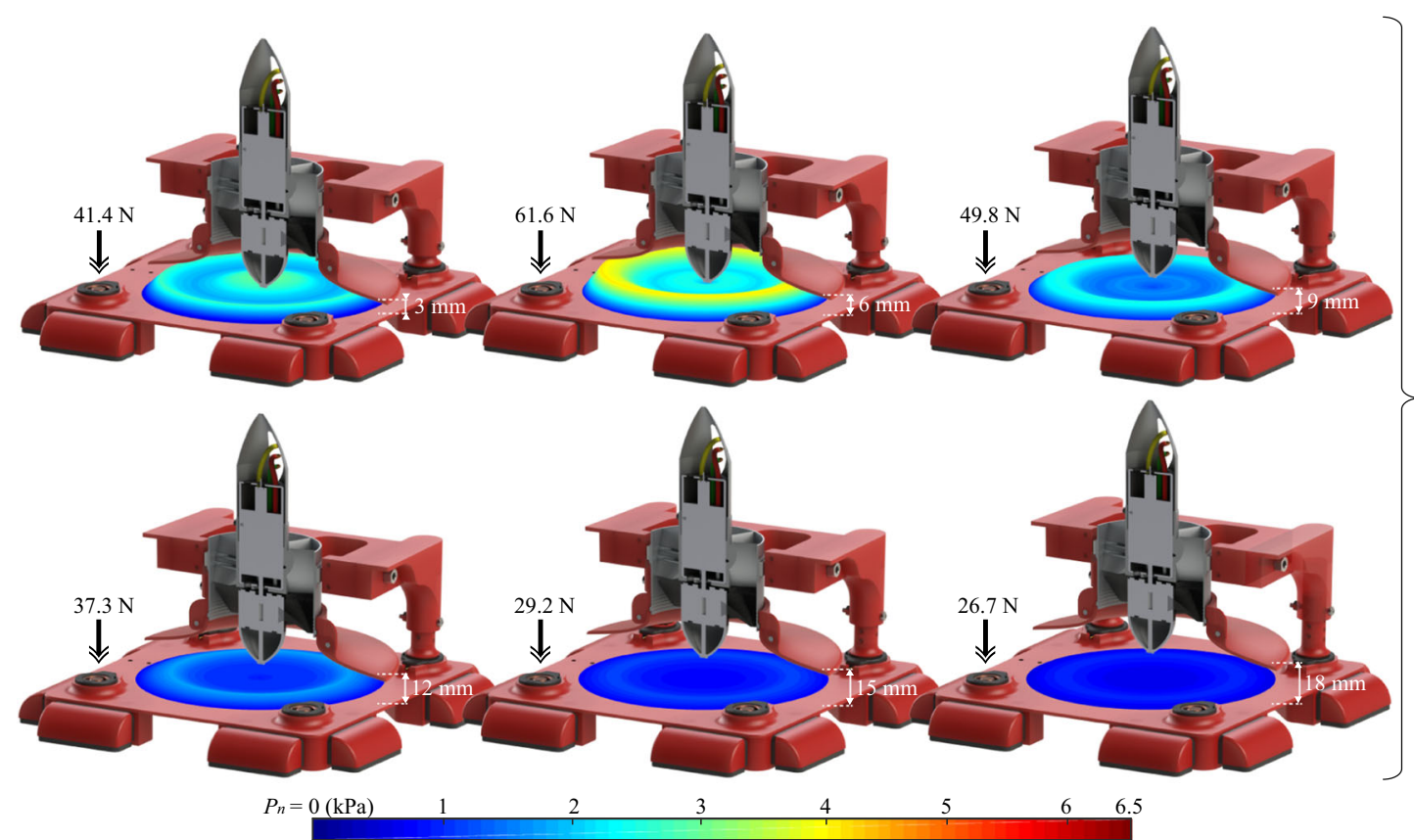

(a)

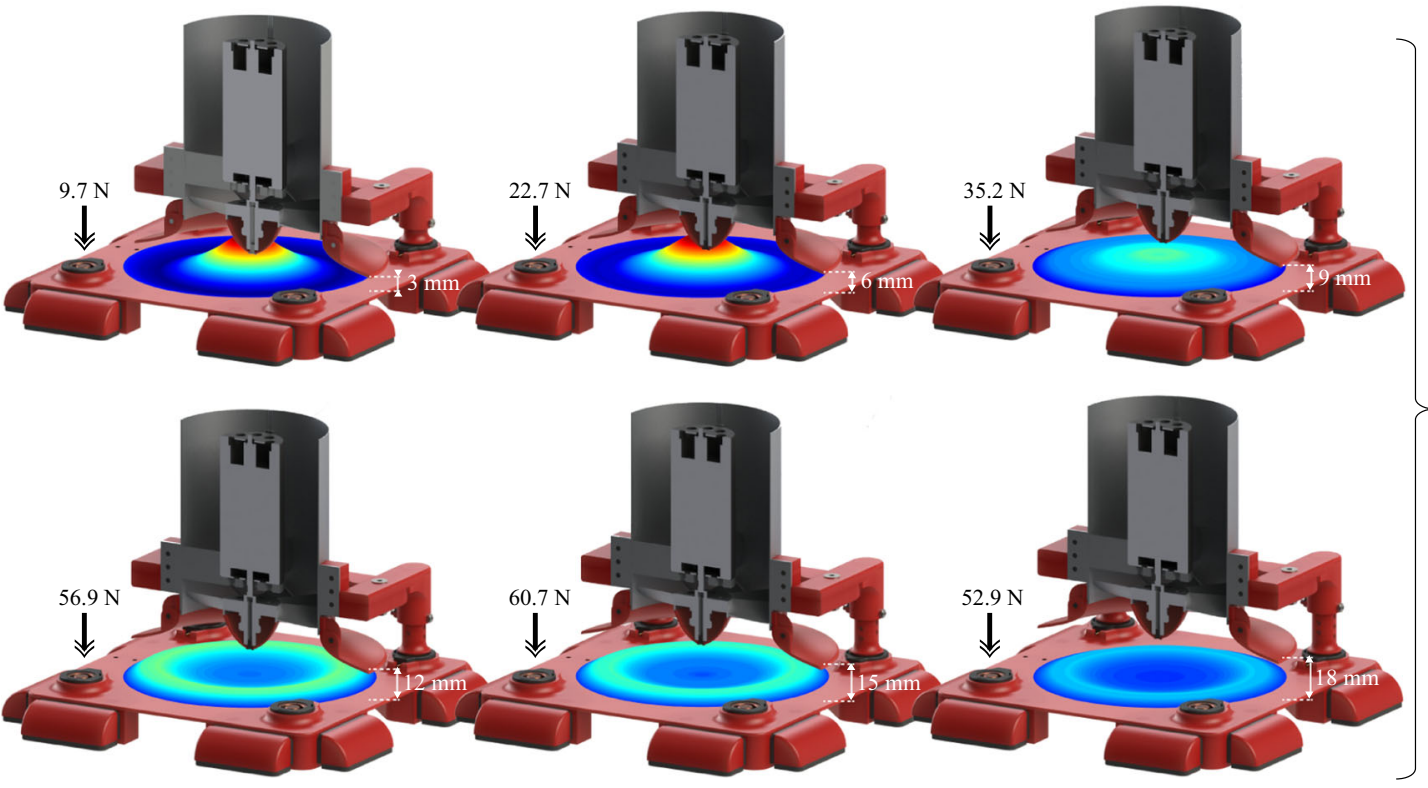

(b)

Fig. 14 Graphical representation of the experimentally acquired pressure distributions on the test surface for the two EDF cases (a-b), under throttle $T=100 \%$, shroud radius $r_{s}=80 \mathrm{~mm}$ and gaps $h=3$ to $18 \mathrm{~mm}$ with $3 \mathrm{~mm}$ increments 
Fig. 15 Test rotors with different number of blades

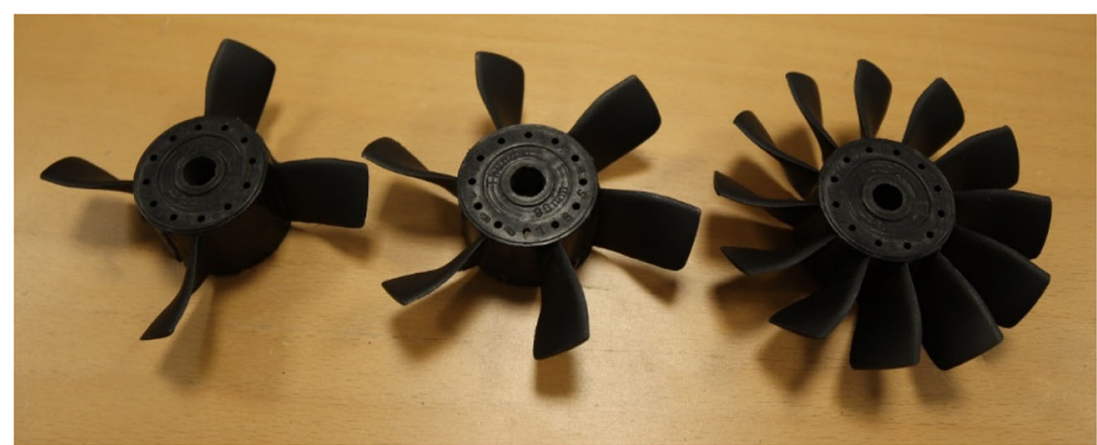

dissipates and gets shifted from the area underneath the duct towards the outer surface area beneath the shroud. This transition starts already from $3 \mathrm{~mm}$ in the case of $\operatorname{EDF}(\mathrm{A})$, while in (B) this transition begins from $9 \mathrm{~mm}$ but progresses much faster.

As the gap increases in (A) and (B) and although the overall negative pressure distribution gets minimized, the force undergoes a big increase for specific gap values. This observation leads to the conclusion that the occurring vortex is not the sole factor for adhesion to the test surface. Specifically, as $h$ increases above a specific threshold, the inflow of air increases suddenly and starts to get propelled through the duct, thus producing thrust. This abrupt change was experimentally observed by a sudden and large increase in sensed flow, which was accompanied by increased noise and vibrations.

The produced thrust results in an additive force component that leads to the observed increase in the measured force summation. Further increase of the gap height would lead to the EDF reaching an adhesion force plateau and generating thrust converging to its free flight equivalent, while the vortex phenomenon is no longer observed in both (A) and (B). This observation provides an important insight to future modeling of these two additive phenomena and presents a novel design strategy for achieving optimal adhesion performance.

\subsection{Additional Design Alterations}

To further investigate the effect of EDF design properties in the VAS adhesion performance, additional experimental trials were performed involving the rotor blade number and the adjustment of the thrust via the addition of an aft duct.

\subsubsection{Robot Blade Alterations}

As documented in EDF-related literature, the number of rotor blades changes significantly the amount of thrust and acquired velocity [18-20]. In free-flight conditions, more blades provide more thrust for the same blade pitch, while the speed decreases and the power consumption has been stated to increase significantly.

For the purpose of evaluating the effect of the rotor's blade number in the case of the VAS and its optimal performance, the EDF (B) was tested with rotors of variable blade number $n_{b}=\{3,4,6,12\}$ (Fig. 15). The experimentally acquired force and power to throttle relationships for the case of $r_{s}=80 \mathrm{~mm}$ and $\mathrm{h}=6 \mathrm{~mm}$ are presented in Fig. 16, where it is evident that the VAS performance decreases with the decrease of blade number, causing a drop in adhesion force of approximately $36.6 \%$ in the case of a 3-blade rotor. The system appears to also have a proportionally lower power consumption,
Fig. 16 Force $F$ and power $P_{c}$ to throttle $T$ hysteresis loops under different rotor blade number for EDF case (B), shroud radius $r_{s}=80 \mathrm{~mm}$ and gap height $h=15 \mathrm{~mm}$
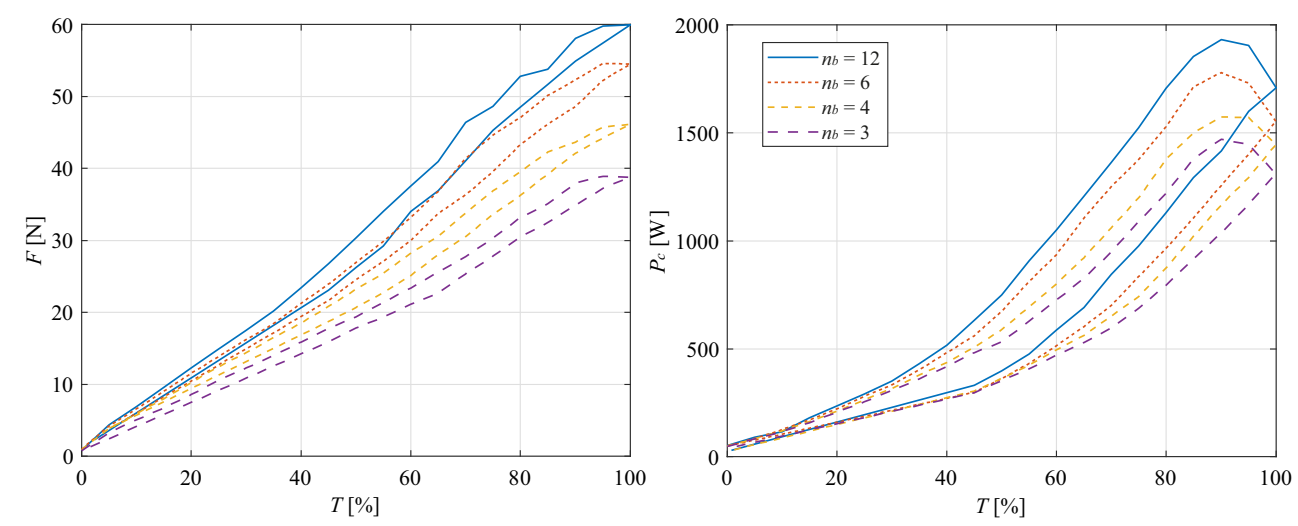
Fig. 17 Aft ducts with alternative outlet areas a Aout,ac $=2 \pi r_{d}^{2}, \mathbf{b}$ Aout $, a c=\mathrm{FSA}, \mathbf{c}$ Aout, $a c=95 \%$ FSA, d Aout, ac $=90 \% \mathrm{FSA}$

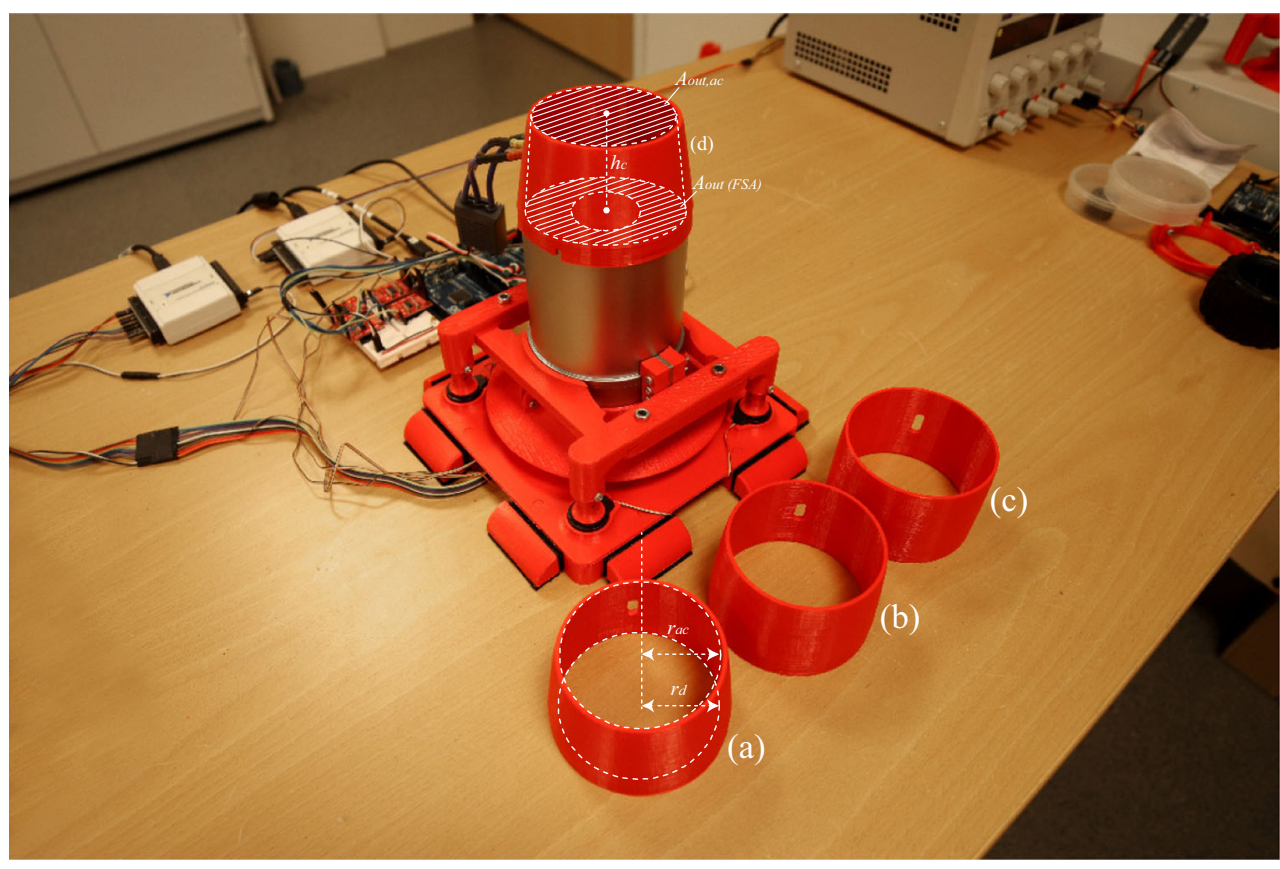

which decreases at approximately $32 \%$. These results agree with the documented free-flight behavior of an EDF when alternating rotor blades.

\subsubsection{Aft Duct}

Another design alteration usually incorporated in EDF designs, with the goal of improving its free-flight characteristics, is the addition of an aft duct to properly adjust the density of the air exiting the back of the EDF. This modification has been traditionally applied in cases where more thrust or speed is needed, as both are affected by the end area of the aft duct. Specifically, it has been documented that an aft duct, with an end area reduced by 5 to $10 \%$ of the FSA (Fan Swept Area-defined as $A_{\text {out }}$ in Section 3), leads to more power/less speed, while for larger decreases up to $20 \%$ it leads to less power/more speed [21]. Drawbacks to this modification have been reported to be the larger power consumption and the reduced cooling to the motor, mainly due to the limitation of the airflow [22].

For the purposes of this evaluation, four different aft ducts were designed and 3D printed for the VAS (B) setup (Fig. 17), where the height $h_{c}$ of all aft ducts has been set to $h_{c}=r_{d}$, while the end area $A_{\text {out }, a c}$ was set to (a) $A_{\text {out }, a c}=2 \pi r_{d}^{2}$, (b) $A_{\text {out }, a c}=\mathrm{FSA}$, (c) $A_{\text {out }, a c}=$ FSA reduced by $5 \%$, and (d) $A_{o u t, a c}=$ FSA reduced by $10 \%$. To experimentally test the aft ducts and evaluate their effect on the VAS and its optimal performance, the EDF (B) was utilized in the case of $r_{s}=80 \mathrm{~mm}$ and $\mathrm{h}=6 \mathrm{~mm}$. The experimentally acquired force and power to throttle relationships are presented in Fig. 18, where it is evident that the VAS performance decreases with the addition of the aft duct and the decrease of its end diameter, causing a drop in adhesion force of approximately $54 \%$ in the case of the
Fig. 18 Force $F$ and power $P_{c}$ to throttle $T$ hysteresis loops under different aft ducts for EDF case (B), shroud radius $r_{s}=80 \mathrm{~mm}$ and gap height $h=15 \mathrm{~mm}$
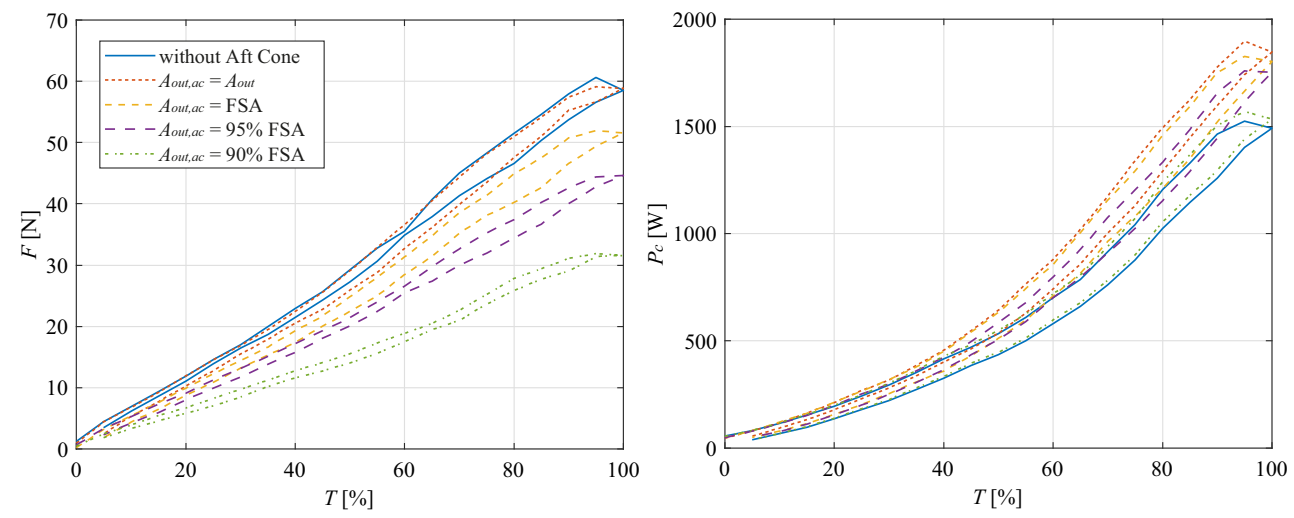
aft duct with end area FSA reduced by $10 \%$. In addition, the system appears to have an increasing power consumption, which reaches an approximate increase of $20 \%$ for the aforementioned case.

\section{Conclusions}

In this article, the negative pressure adhesion properties of an Electric Ducted Fan were experimentally evaluated. The goal of this study was the structural optimization of the EDF's adhesion efficiency for its future incorporation in a Wall Climbing Robot (WCR). To this goal, a Vortex Actuation System (VAS) was designed and developed for measuring the pressure distribution and adhesion force generated when an EDF is placed at close proximity to a test surface. Variable design characteristics regarding shroud radii, distances from the target surface, rotor blade number and elongation of the duct were investigated to determine their structural impact on the generated adhesion force responses.

Experimental trials were focused on shroud radius and distance from surface (gap), which led to the important observation that an increase in gap height for the same shroud radius leads to a significant decrease in negative pressure, but the force does not follow the same decreasing behaviour. This revealed the additive nature of a thrust force component, which was occurring abruptly after exceeding a specific gap threshold and was leading to large increase in adhesion efficiency. The results involving the maximum forces acquired for both EDF cases and all combinations of shroud radius and gaps showed an optimal point of operation for specific combinations of shroud radius and gap, which when compared to the two EDF free-flight maximum thrusts led to a maximum increase of $149 \%$ and $58 \%$ in exerted force, respectively.

A correlation was investigated that would describe the relationship of the EDF inherent dimensions (duct diameter, motor diameter etc.), the variable dimensions (shroud diameter, gap) to the maximum acquired forces. The plotting of forces to the ratio of the inlet and outlet areas of the EDF showed a convergence of optimum performances to the same value for both EDFs. This finding was deemed of particularly high importance, since it determines the design details of all future prototypes incorporating the VAS, as trade-offs between EDF dimensions, shroud size and gap are introduced. As the optimal adhesion force was approximately the same $(60 \mathrm{~N})$ for both cases, it would lead the EDF choice down to the overall performance, power consumption and robustness of the system. This choice will be ultimately based to a series of trade-offs and it is a matter that needs further investigation.

The increase in the EDF throttle caused a clear increase in the negative pressure for both cases, which became progressively greater for the points closer to the surface center, thus revealing the generation of a vortex. An insight was given on the pressure distribution during the VAS operation in different configurations, where an increasing behaviour was observed as the shroud radius increased and indicated a clear change in pressure distribution as the gap altered for both EDF cases. These results showed an evident dissipation of the vortex phenomenon and the generation of a ripple-like pressure distribution on the surface points enclosed by the shroud.

Another research objective that was created from the presented findings is the need to maximize the performance while minimizing the power consumption, which in the case of EDF-based designs can reach very high values. The acquired hysteresis information on the power consumption will be used to develop optimal control algorithms to ensure that the VAS system provides the necessary adhesion performance for the robotic platform to remain attached on the surface while the consumption remains as small as possible. This objective becomes even more important in the design case of incorporating multiple VAS actuators in the WCR, where the consumption will play an intricate role in the overall performance.

Finally, to further investigate the effect of EDF design properties in the VAS adhesion performance, additional experimental trials were performed involving the rotor blade number and the adjustment of the thrust via the addition of an aft duct. Experiments via reducing the rotor blade number revealed a decrease in both adhesion performance and consumption, while the experiments involving aft ducts of different outlet areas displayed a decrease in adhesion performance and increase in consumption.

The presented study and produced results will act as a new knowledge basis on EDF-based designs and the cornerstone for the modeling and control of this setup, with the goal of incorporating the optimized negative pressurebased actuator in a WCR for inspection and repair of airplane fuselages.

Funding Information This work has received funding from the European Union's H2020 Framework Programme under the call FETOPEN, grant agreement No. 665238.

\section{Compliance with Ethical Standards}

Conflict of Interest The authors declare that they have no conflict of interest.

Open Access This article is distributed under the terms of the Creative Commons Attribution 4.0 International License (http://creativecommons. org/licenses/by/4.0/), which permits unrestricted use, distribution, and reproduction in any medium, provided you give appropriate credit to the original author(s) and the source, provide a link to the Creative Commons license, and indicate if changes were made.

Publisher's Note Springer Nature remains neutral with regard to jurisdictional claims in published maps and institutional affiliations. 


\section{References}

1. Brusell, A., Andrikopoulos, G., Nikolakopoulos, G.: Novel considerations on the negative pressure adhesion of electric ducted fans: an experimental study. In: 25th Mediterranean Conference on Control and Automation (MED), Valletta (2017)

2. Abrego, B.R.W., Anita, I., Anita, I.: Performance study of a ducted fan system. In: American Helicopter Society Aerodynamics, Acoustics, and Test and Evaluation Technical Specialists Meeting, San Francisco (2002)

3. Xiao, J.X.J., Sadegh, A., Elliott, M., Calle, A., Persad, A., Chiu, H.M.C.H.M.: Design of mobile robots with wall climbing capability. In: Proceedings, 2005 IEEE/ASME International Conference on Advanced Intelligent Mechatronics, pp. 24-28 (2005)

4. Xiao, J., Li, B., Ushiroda, K., Song, Q.: Rise-rover: a wallclimbing robot with high reliability and load-carrying capacity. In: Proceedings of the 2015 IEEE Conference on Robotics and Biomimetics, pp. 2072-2077 (2015)

5. Brusell, A., Andrikopoulos, G., Nikolakopoulos, G.: A survey on pneumatic wall-climbing robots for inspection. In: 24th Mediterranean Conference on Control and Automation (MED), 21-24 June. Athens, Greece (2016)

6. Kawasaki, S., Kikuchi, K.: Development of a small legged wall climbing robot with passive suction cups. In: The 3rd International Conference on Design Engineering and Science, ICDES 2014, pp. 112-116 (2014)

7. Yoshida, Y., Ma, S.: Design of a wall-climbing robot with passive suction cups. In: 2010 IEEE International Conference on Robotics and Biomimetics, pp. 1513-1518 (2010)

8. Shang, J., Sattar, T., Chen, S., Bridge, B.: Design of a climbing robot for inspecting aircraft wings and fuselage. Ind. Robot: Int. J. 34(6), 495-502 (2007)

9. Pack, R.: A rubbertuator-based structure-climbing inspection robot. In: IEEE International Conference on Robotics and Automation, no. April, pp. 1869-1874 (1997)

10. Zhou, Q., Li, X.: Design of wall-climbing robot using electrically activated rotational-flow adsorption unit. In: 2016 IEEE/RSJ International Conference on Intelligent Robots and Systems (IROS), pp. 5758-5763 (2016)

11. Zhang, Y., Dodd, T., Atallah, K., Lyne, I.: Design and optimization of magnetic wheel for wall and ceiling climbing robot. In: 2010 IEEE International Conference on Mechatronics and Automation, pp. 1393-1398 (2010)

12. Bi, Z., Guan, Y., Chen, S., Zhu, H., Zhang, H.: A miniature biped wall-climbing robot for inspection of magnetic metal surfaces. In: 2012 IEEE International Conference on Robotics and Biomimetics (ROBIO), pp. 324-329 (2012)

13. Degani, A.: Dynamic single actuator robot climbing a chute: period-doubling bifurcations: analysis and experiments. Meccanica 51(5), 1227-1243 (2016)

14. Yan, Y., Liu, Y., Páez Chávez, J., Zonta, F., Yusupov, A.: Proofof-concept prototype development of the self-propelled capsule system for pipeline inspection. Meccanica 1-16 (2017)

15. Sekhar, P., Bhooshan, R.: Duct fan based wall climbing robot for concrete surface crack inspection. In: IEEE India Conference (INDICON) (2014)
16. Guan, Y., Zhu, H., Wu, W., Zhou, X., Jiang, L., Cai, C., Zhang, L., Zhang, H.: A modular biped wall-climbing robot with high mobility and manipulating function. IEEE/ASME Trans. Mechatron. 18(6), 1787-1798 (2013)

17. Sharman, R.A.: Electric ducted fan theory and practice. [Online]. Available: http://www.rcgroups.com/forums/showatt. php?attachmentid=6384145 (2013)

18. Turner, R.C.: Notes on ducted fan Design. Gt. Britain Aeronautical. Research Council (1964)

19. Raymer, D.P.: Aircraft design: a conceptual approach (4th edn), AIAA Education series, California-USA, 2006, ISBN, 1-56347829-3, 840p

20. Ko, A., Ohanian, O.J., Gelhausen, P.: Ducted fan UAV modeling and simulation in preliminary design. In: AIAA Modeling and Simulation Technologies Conference and Exhibit, August 2007, AIAA 2007-6375 (2007)

21. Electric Ducted Fan Theory. [Online]. Available: http://www.rcex. cz/kestazeni/EDF_THR3.pdf

22. Shima, J.: Introduction to EDF (Electric Ducted Fan) model flying. AAM Meeting Presentation, 2011. [Online]. Available: http://www.hyperdynelabs.com/RC/AAM/EDF/Introduction $\% 20$ of $\% 20$ EDF\%20(Electric\%20Ducted\%20Fan).ppt

George Andrikopoulos received his Ph.D degree in the field of Automatic Control Engineering from the University of Patras, Greece, in 2015. He is currently working as a post-doctoral researcher with the Department of Computer Science, Electrical and Space Engineering at the Luleå University of Technology, Sweden. His current research interests are mainly focused on the design, development and control of biorobotic systems via the use of soft pneumatic actuators, as well as robotic solutions for inspection and home service purposes. $\mathrm{He}$ has been involved in a number of European and National projects on biorobotics, inspection and industrial robotics including H2020 FETOPEN RIA "CompInnova", Swedish Research Council (Vetenskapsrådet) "BAHRT", as well as research collaborations with Honda Research Institute Japan and Swedish Universities and Colleges. He has served as a reviewer for several international journals and conferences and he is a member of IEEE, CSS, RAS, IES, SPS.

George Nikolakopoulos received the Ph.D. degree from the Department of Electrical and Computer Engineering, University of Patras, Rio, Greece, in 2006.

$\mathrm{He}$ is an Associate Professor with the Control Engineering Group, Division of Systems and Interaction, Faculty of Automatic Control Systems, Luleå University of Technology, Luleå, Sweden. His published scientific works include more than 90 published papers in international journals and conference proceedings in his fields of interest. His main research interests include fields such as networked controlled systems, mechatronics, wireless sensor networks and actuators, AUVs, UAVs, robotics, adaptive control, and system identification. He was a project manager in several R\&D projects funded by the EU, ESA, and Greek National Ministry of Research. He received the Information Societies Technologies (IST) Prize Award for the best paper that promotes the scopes of the European IST (currently known as ICT) in 2003. 